\title{
Increased replication of dissimilatory nitrate-reducing bacteria leads to decreased anammox bioreactor performance
}

\author{
Ray Keren ${ }^{1 \dagger}$, Jennifer E. Lawrence ${ }^{2 \dagger}$, Weiqin Zhuang $^{3}$, David Jenkins ${ }^{1}$, Jillian F. Banfield ${ }^{4}$, Lisa Alvarez-Cohen ${ }^{1,5}$, \\ Lijie Zhou ${ }^{6 *}$ and $\mathrm{Ke} \mathrm{Yu}^{7^{*}}$ (D)
}

\begin{abstract}
Background: Anaerobic ammonium oxidation (anammox) is a biological process employed to remove reactive nitrogen from wastewater. While a substantial body of literature describes the performance of anammox bioreactors under various operational conditions and perturbations, few studies have resolved the metabolic roles of their core microbial community members.

Results: Here, we used metagenomics to study the microbial community of a laboratory-scale anammox bioreactor from inoculation, through a performance destabilization event, to robust steady-state performance. Metabolic analyses revealed that nutrient acquisition from the environment is selected for in the anammox community. Dissimilatory nitrate reduction to ammonium (DNRA) was the primary nitrogen removal pathway that competed with anammox. Increased replication of bacteria capable of DNRA led to the out-competition of anammox bacteria, and the loss of the bioreactor's nitrogen removal capacity. These bacteria were highly associated with the anammox bacterium and considered part of the core microbial community.

Conclusions: Our findings highlight the importance of metabolic interdependencies related to nitrogen- and carbon-cycling within anammox bioreactors and the potentially detrimental effects of bacteria that are otherwise considered core microbial community members.
\end{abstract}

Keywords: Anammox, Metagenomics, Metabolism, Dissimilatory nitrate reduction (DNRA), Nitrogen cycle

\section{Background}

Anaerobic ammonium oxidizing (anammox) bacteria obtain energy from the conversion of ammonium and nitrite to molecular nitrogen gas $\left(\mathrm{N}_{2}\right)$ [1]. Currently, the only bacteria known to catalyze this process are members of the phylum Planctomycetes [2, 3], none of which have been isolated [3, 4]. In practice, anammox bacteria are employed in an eponymous process in combination with the partial nitritation $(\mathrm{PN})$ process to remove ammonium from nitrogen-rich

\footnotetext{
*Correspondence: pakerzhou@szu.edu.cn; yuke.sz@pku.edu.cn

${ }^{\dagger}$ Ray Keren and Jennifer E. Lawrence share senior authorship.

${ }^{6}$ College of Chemistry and Environmental Engineering, Shenzhen University, Shenzhen, China

${ }^{7}$ School of Environment and Energy, Shenzhen Graduate School, Peking University, Shenzhen, GD, China

Full list of author information is available at the end of the article
}

wastewaters. First, in PN, approximately half of the ammonium in solution is aerobically oxidized to nitrite. Second, in anammox, both ammonium and nitrite are anaerobically converted to $\mathrm{N}_{2}[5,6]$. The PN/anammox (i.e., deammonification) process is beneficial because it consumes $60 \%$ less energy, produces $90 \%$ less biomass, and emits a significantly smaller volume of greenhouse gas than conventional nitrogen removal by nitrification and denitrification processes [7]. To date, over 100 full-scale deammonification process bioreactors have been installed at municipal and industrial wastewater treatment plants across the globe [8].

Within an engineered environment, anammox bacteria have very low growth rates and can easily become inhibited by fluctuating substrate and metabolite concentrations $[9,10]$. When these two limitations are coupled,

(c) The Author(s). 2020 Open Access This article is distributed under the terms of the Creative Commons Attribution 4.0 International License (http://creativecommons.org/licenses/by/4.0/), which permits unrestricted use, distribution, and 
recovery from an inhibition event can take up to 6 months (which is unacceptably long for municipalities that must meet strict nitrogen discharge limits) [11]. Furthermore, these problems are compounded by a cursory understanding of the microbial communities that exist alongside anammox bacteria. A deeper understanding of the complex interactions occurring among bacterial species in an anammox bioreactor is required for the broad application of the deammonification process for wastewater treatment.

Previous research has suggested that a core microbial community exists within anammox bioreactors [12-16]. In the majority of studied bioreactors, uncultured members of the phyla Bacteroidetes, Chloroflexi, Ignavibacteria, and Proteobacteria have been identified alongside Planctomycetes, the phylum that contains anammox bacteria. These phyla have primarily been identified through $16 \mathrm{~S}$ rRNA gene studies, so their interplay with anammox performance has not yet been fully elucidated [12-16]. From their taxonomic identity and performance studies, it is assumed that the additional phyla compete for nitrite and cooperate to transform (i.e., dissimilatory nitrate reduction to ammonium; DNRA) and remove (i.e., denitrifiers) nitrate, a product of anammox metabolism [17-19].

Here, we illuminate deeper metabolic relationships between an anammox bacterium, Brocadia, and its supporting community members during the start-up and operation of a laboratory-scale anammox bioreactor. We begin by analyzing the formation of the "anammox community" through a combination of genome-centric metagenomics and 16S rRNA gene sequencing. Metabolic features of positively enriched bacteria are compared to negatively enriched bacteria during the start-up process. Next, we focus our investigation on an anammox performance destabilization event that was driven by microbial interactions. Last, we conduct a comparative analysis of our anammox community to similarly studied anammox communities $[18,20]$ to highlight the broader relevance of our results. To our knowledge, this is the first time series-based study to link anammox metagenomic insights and community composition to anammox bioreactor functionality [21]. Our findings bolster the fundamental, community-level understanding of the anammox process. Ultimately, these results will enable more comprehensive control of this promising technology and facilitate its widespread adoption at wastewater treatment plants.

\section{Results}

\section{Bioreactor performance}

The performance of a laboratory-scale anammox anaerobic membrane bioreactor (MBR) (described in the "Methods" section) was tracked for 440 days from initial inoculation, through several performance crashes, to stable and robust anammox activity (Fig. 1). Performance was quantified by nitrogen removal rate (NRR; g-N
$\mathrm{L}^{-1} \mathrm{~d}^{-1}$ ) and effluent quality (g- $\left.\mathrm{N} \mathrm{L}^{-1} \mathrm{~d}^{-1}\right)$. Bioreactor performance generally improved over the first 103 days of operation. At this point, the hydraulic residence time (HRT) was reduced from 48 to $12 \mathrm{~h}$ and influent nitrogen concentrations were reduced to maintain a stable loading rate. Additional seed biomass from a nearby pilot-scale deammonification process was added on Day 145 following a performance crash and bioreactor performance improved, enabling influent ammonium and nitrite concentrations to be steadily increased until the NRR approached $2 \mathrm{~g}-\mathrm{N} \mathrm{L}^{-1} \mathrm{~d}^{-1}$. On Day 189, the bioreactor experienced a technical malfunction and subsequent performance crash, identified by a rapid decrease in the NRR and the effluent quality. On Day 203, the bioreactor was again amended with a concentrated stock of seed biomass and the NRR and the effluent quality quickly recovered. Influent ammonium and nitrite concentrations were again increased until the NRR reached $2 \mathrm{~g}-\mathrm{N} \mathrm{L}^{-1} \mathrm{~d}^{-1}$.

The bioreactor subsequently maintained steady performance for approximately 75 days, until Day 288, when effluent concentrations of ammonium and nitrite unexpectedly began to increase and nitrate concentrations disproportionately decreased. Seven days later, the NRR rapidly plummeted. No technical malfunctions had occurred, indicating that the destabilization of the anammox process may have been caused by interactions among its microbial community members. At the time, the cause of the performance decline was not understood, so the bioreactor was not re-seeded with biomass. After 50 days of limited performance, concentrations of copper, iron, molybdenum, and zinc in the bioreactor influent were increased based on literature recommendations [22-25] and the NRR rapidly recovered. Stable and robust bioreactor performance was subsequently maintained.

\section{Metagenomic sequencing and binning}

Whole community DNA was extracted and sequenced at six time points throughout the study: Day 0 (D0), for inoculant composition; Day 82 (D82), during nascent, positive anammox activity; Day 166 (D166), 3 weeks after an additional biomass amendment; Day 284 (D284), after a long period of stable and robust anammox activity and just before the bioreactor performance was destabilized; Day 328 (D328), in the midst of the performance destabilization period; and Day 437 (D437), during mature, stable, and robust anammox activity.

From all samples, 337 genomes were binned, 244 of which were estimated to be $>70 \%$ complete by checkM [26]. The genomes were further dereplicated across the six time points into clusters at $95 \%$ average nucleotide identity (ANI). This resulted in 127 representative and unique genomes (Additional file 1: Table S1) that were used for all downstream analyses. Mapping showed an 


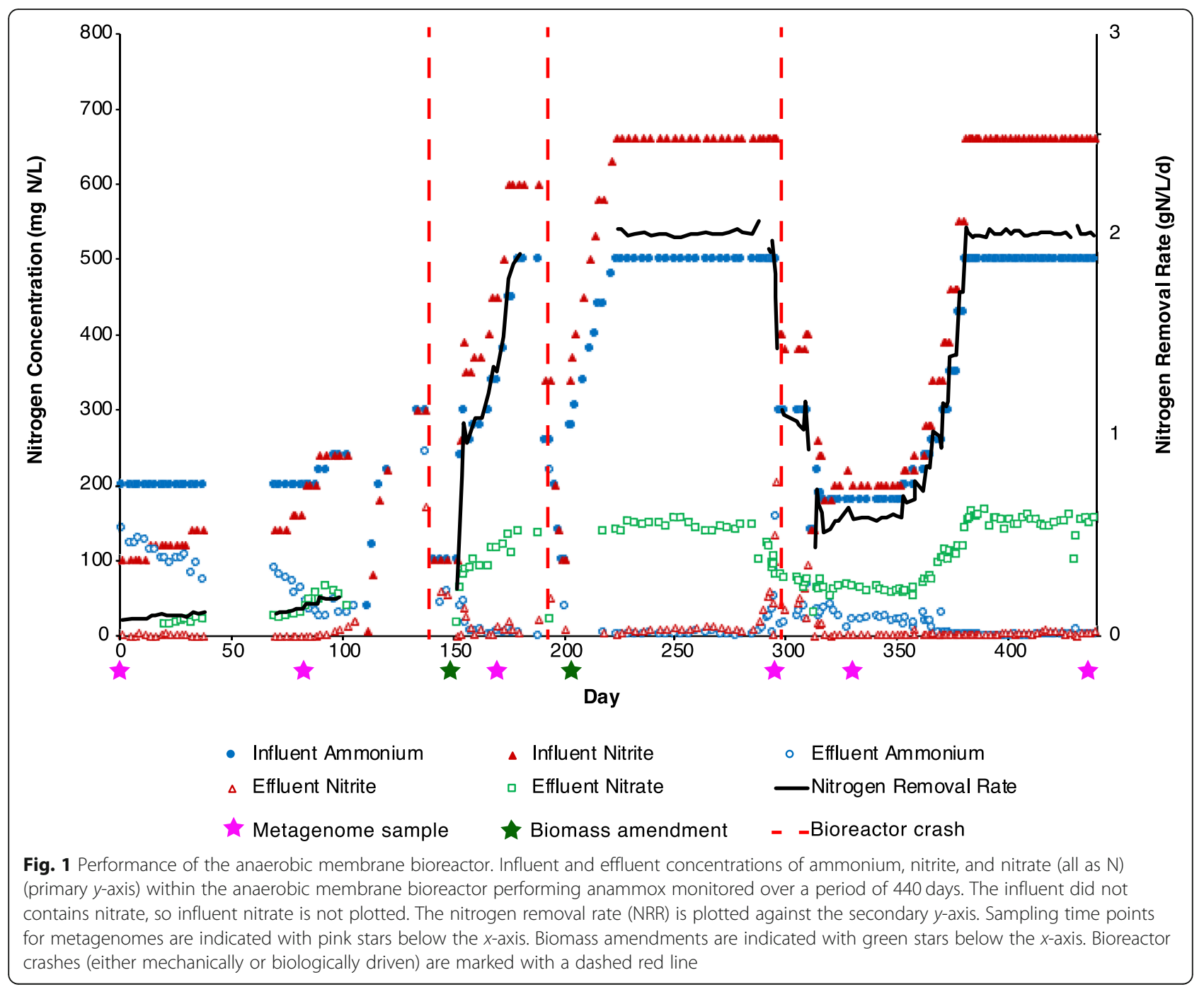

average read recruitment of $76 \%$ to the representative genomes (Table 1). The number of genomes present at each time point (using threshold values of coverage $>1$ and breadth >0.5) ranged from 60 (D437) to 103 (D166). In addition, nine strains were detected that differed from the representative genome by $2 \%$ ANI (Additional file 1: Table S2). Except for the anammox bacterium, which is referenced at the genus level
(Brocadia), all representative genomes are referenced at the phylum level.

\section{Community structure and temporal dynamics}

As both internal and external factors can work in combination to affect the structure of a bioreactor community, we hypothesized that different groups of bacteria (i.e., sub-communities) would be associated with

Table 1 Read counts mapped to representative genomes across time points

\begin{tabular}{lllll}
\hline Time point (days) & Total read number & Mapped read number & \% of reads mapped & Number of genomes detected* $^{*}$ \\
\hline 0 & 55398280 & 40291503 & 72.73 & 92 \\
82 & 62544544 & 43877427 & 70.15 & 82 \\
166 & 60931806 & 46030350 & 75.54 & 103 \\
284 & 56282006 & 48644523 & 86.43 & 68 \\
328 & 127048582 & 95132145 & 74.88 & 87 \\
437 & 119945232 & 93737087 & 78.15 & 60
\end{tabular}

"Number of representative genomes based on threshold of $>1$ coverage and $>0.5$ breadth 
different phases of the bioreactor's lifespan. To test for grouping, all genomes were pairwise-correlated (Fig. 2a). The resulting heatmap revealed four distinct clusters (Groups A-D). Group A was the largest, with 52 genomes, while Groups B-D had 25, 24, and 26 genomes, respectively (Additional file 1: Table S3).

To better examine the clustering of genomes in relation to the bioreactor's lifespan, we ran nonmetric multidimensional scaling (nMDS) analyses on the genomes' relative abundance data (Fig. $2 \mathrm{~b}$ ). The nMDS projection revealed that genome groups were strongly associated with specific time points: Group A was associated with the inoculant biomass at D0 and D166, while Group C was associated with the nascent anammox community at D82. Group B was associated with the times of destabilized anammox performance (Days 284-328), and Group $\mathrm{D}$ was associated with the mature, stable anammox community at D437. Brocadia is a part of Group D, although its location on the nMDS projection is skewed to the left because of its high relative abundance throughout the majority of the bioreactor's lifespan. Because the nascent anammox community was amended with additional biomass, we could not resolve a linear trajectory for the microbial community between the initial and final states. Nevertheless, Groups B and D shared many similarities, and the majority of the genomes associated with Group B were still present in the bioreactor on D437.
To further resolve the relative abundances of Groups A-D over the bioreactor's lifespan, 16S rRNA genes from genomes were combined with direct $16 \mathrm{~S}$ rRNA sequencing data, then clustered into operational taxonomic units (OTUs). Of the 127 representative genomes, 34 contained a $16 \mathrm{~S}$ rRNA sequence that was successfully clustered to an OTU. The matching OTUs represented $55 \%$ of the total $16 \mathrm{~S}$ rRNA reads at Day 9, but quickly increased to an average representation of $86 \%$. The OTUs that match assembled genomes were grouped (Fig. 2) and their relative abundance summed (Fig. 3a). The matching genomes comprised $18 / 52,10 / 25,3 / 24$, and $7 / 26$ of Groups A-D, respectively. The matching OTUs represented $55 \%$ of the total 16S rRNA reads at Day 9, but quickly increased to an average representation of $86 \%$.

Group A was dominant on Day 0 but rapidly decreased in abundance by the first $16 \mathrm{~S}$ rRNA gene sequencing time point on Day 9 (Fig. 3b). Group A was again dominant after the addition of new inoculum on Day 145 (Fig. 3a). Group B (and to a certain extent Group A) became dominant just before Day 300 when the anammox performance was destabilized.

To check the accuracy of the 16S rRNA matching to the metagenomic data, we compared the relative abundances of Groups A-D across three data sub-sets (Fig. 3b): all metagenomes (marked "met" on the $x$-axis), only metagenomes with matching $16 \mathrm{~S}$ rRNA OTUs (marked "sub-
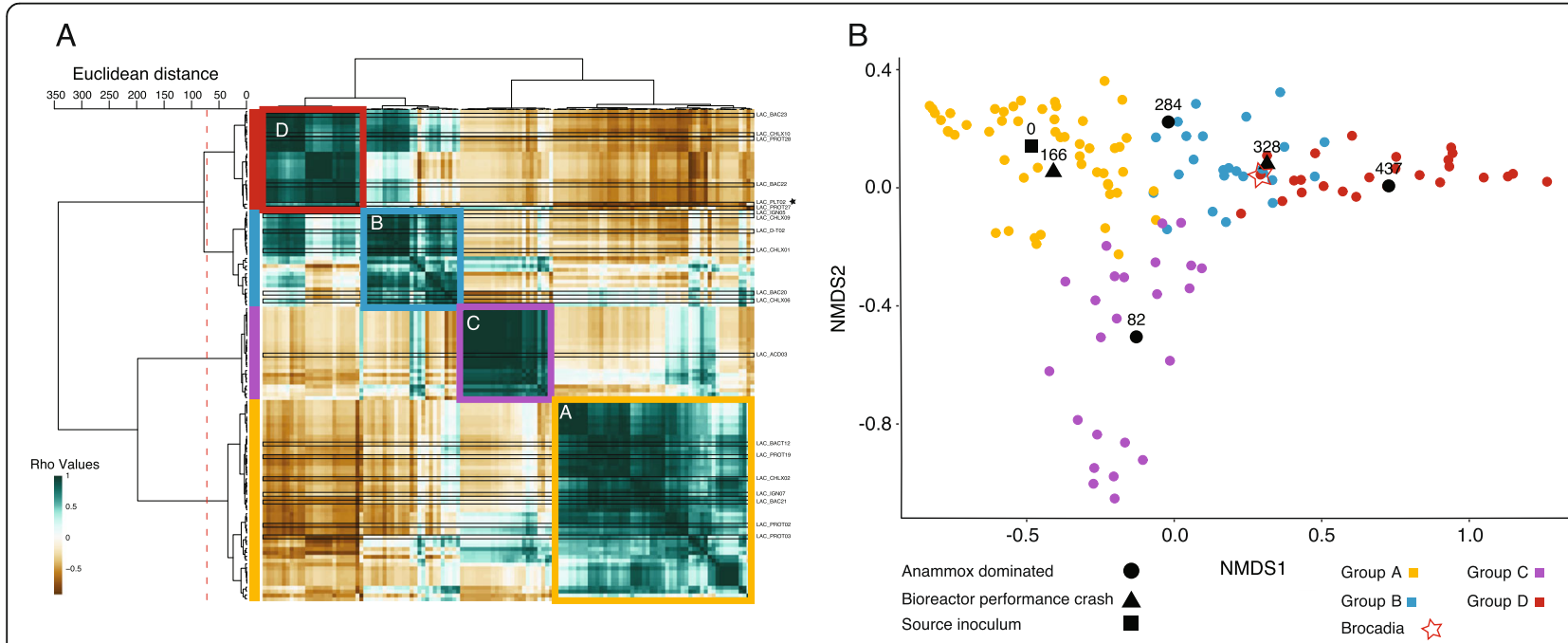

Fig. 2 Analysis of the bioreactor's community clustering, using the relative abundance of the bacteria. a clustering heatmap of bacteria based on pairwise cross correlations for the six time points (matrix values are Rho values). Color scales mark high positive correlation in green and negative correlation in brown. The row and column dendrograms are identical (consisting of the genomes). The row dendrogram shows the calculated distance between the clusters with a dashed red line marking the split into clusters. Colored squares as well as bars to the left of the heat map show relative abundance-based grouping: Yellow indicates Group A; blue, Group B; purple, Group C; and red, Group D. A black star to the right of the heatmap marks the anammox bacterium (Brocadia). b Two-dimensional nMDS projection of bacteria and time points, showing the association of the bacteria (and relative abundance groups to certain time points). Each colored dot represents the centroid of a bacterium, with colors matching the relative abundance group. Black marks represent the centroid of the time points, while the shape represents the state of the bioreactor: circle indicates anammox dominated; triangle, bioreactor crash (either mechanical or biological; and square, time zero. The location of Brocadia is marked with a red star 
A

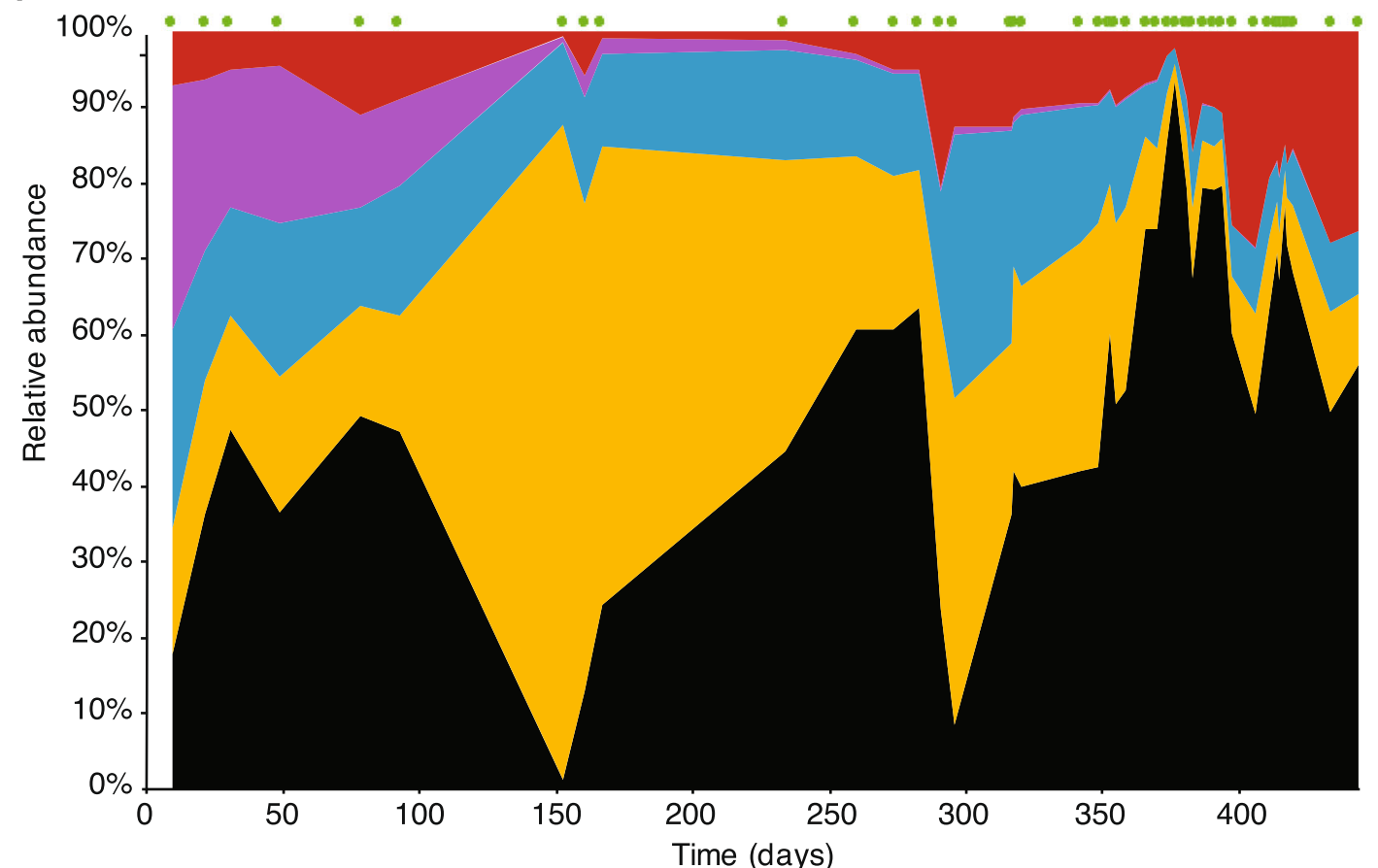

B

B Anammox $\square$ Group A $\square$ Group B $\square$ Group C $\square$ Group D $\square$ Sampling point $\odot$

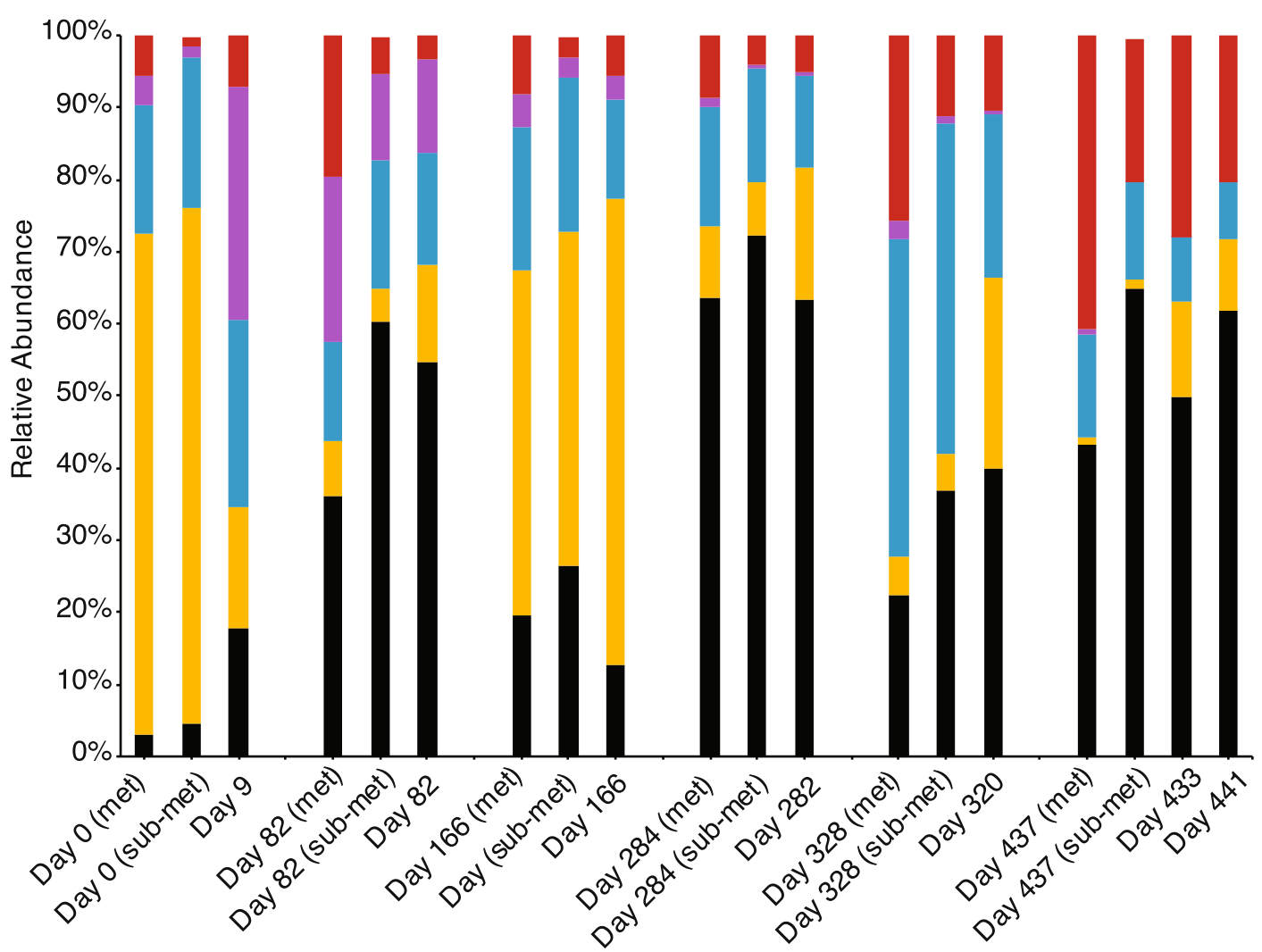

Fig. 3 (See legend on next page.) 
(See figure on previous page.)

Fig. 3 Relative abundances of bacterial groups over the lifespan of the bioreactor. a Relative abundances of bacterial Groups A-D, based on 165 rRNA OTUs that have a match to a draft genome. Group colors are matched by the analysis in Fig. 2, with the exception of Brocadia, which was removed from Group D and is depicted in black. Green points above the graph show timepoints where the community was sampled. $\mathbf{b}$ Comparison of relative abundances of the different groups at the same time (or the closest time) across sequencing platforms. Relative abundance was calculated for groups based on all the draft genomes (marked met on the $x$-axis), the subset of genomes with matched to the $16 \mathrm{~S}$ rRNA OTUs (marked sub-met on the $x$-axis) or the $16 \mathrm{~S}$ rRNA OTUs (only day is marked on $x$-axis)

met" on the $x$-axis), and the 16S rRNA OTUs. Overall, the three datasets were compatible, with slight variations in over/under-estimations of particular groups. In comparison to the metagenomically derived data, the $16 \mathrm{~S}$ rRNA data tended to overestimate the relative abundance of Group A and underestimate the relative abundance of Group D. A large fraction of the Chloroflexi in Group D was not matched to $16 \mathrm{~S}$ rRNA OTUs, so the underestimation was consistent with expectations.

For all subsequent analyses, we split the representative genomes into two groups (Additional file 1: Table S3): those that are associated with the mature anammox community at D437 (anammox associated, AA) and those that are not (source associated, SA). The AA community includes all of the genomes that are present at D437, while the SA community includes the rest of the genomes that are not present at D437. Some of these genomes are associated with the sludge amendments, and some are associated with the nascent anammox community; at no point is there a community exclusively comprised of SA genomes.

\section{Metabolic profiles}

For the purpose of analyzing the metabolic potential of the microbial community, we evaluated only genomes with $>70 \%$ completeness $(n=88)$ [26]. Using Hidden Markov Model (HMM) searches of the Kyoto Encyclopedia of Genes and Genomes (KEGG) database, we checked for the presence of genes based on their KEGG Orthology (KO) number and calculated KEGG module completeness $[27,28]$. The genomes were clustered by $\mathrm{KO}$ presence/absence (Additional file 1: Figure S1) and their module completeness (Fig. 4). Clustering by the two methods resulted in similar groupings.

Module clustering resolved five groups $(a, \beta, \gamma, \delta, \varepsilon)$ (Fig. 4a and Additional file 1: Table S3). Groups a and $\beta$ contained more anammox-associated genomes $(90 \%$ and $60 \%$, respectively), while Groups $\gamma, \delta$, and $\varepsilon$ contained $65 \%, 70 \%$, and $60 \%$ of source-associated genomes, respectively. The clustering was also strongly influenced by bacterial taxonomy (Fig. 4b). Group a was composed solely of Gram (+) bacteria, primarily Chloroflexi. Group $\beta$ was composed of Candidate Phyla Radiation (CPR) bacteria, Microgenomates. This group of bacteria has reduced genomes and metabolism [29] and thus has an unknown effect on the community metabolism. Group $\mathrm{y}$ was composed entirely of Gram (-) bacteria from a wide range of phyla and includes the anammox bacterium Brocadia. Group $\delta$ was composed of Ignavibacteria and Bacteroidetes, but only the Ignavibacteria from Group $\delta$ were associated with the AA group. Accordingly, further analysis of Group $\delta$ refers only to the Ignavibacteria. Group $\varepsilon$ was composed entirely of Proteobacteria.

Based on the KEGG module clustering, we reconstructed the representative metabolisms of the five groups (Fig. 5). We used a module completeness threshold of $67 \%$ per genome and considered it representative if it was complete in $>50 \%$ of the group's members. Group $\delta$ was not represented since it diverged from Group $\gamma$ by auxotrophies in several modules (Fig. 4a, red rectangle). The Brocadia metabolism is shown in Additional file 1: Figure S2.

While module completeness was used for most of the analyses, in several cases it was not sufficient (e.g., overlap between modules, no module for path). For oxidative phosphorylation, fermentation, carbon fixation, several amino acid synthesis pathways, and nitrogen metabolism, we analyzed gene presence manually. For anammox, four additional HMMs were added: hydrazine synthase subunit A ( $h z s A)$, hydrazine oxidoreductase subunit A $(h z o A)$, and nitrite oxidoreductase subunits $n r x A$ and $n r x B$ [30]. For the latter, the similarity of the gene to the nitrate reductase narGH was taken into consideration.

With the exception of two CPR bacteria, all of the genomes in the bioreactor contained genes encoding assimilation of ammonia into glutamate (Fig. 6). More than half (49) of the bacteria could potentially reduce nitrate, and the same number had the genes needed to further reduce nitrite to nitrogen monoxide (NO); however, only 26 bacteria had the genes to do both steps. The remaining steps of denitrification were encoded in an even smaller number of genomes. The $n r x A B$ gene was only identified in two genomes, one of which was Brocadia. One-step DNRA was identified in 22 genomes. While the number of genes encoding ammonia assimilation and nitrate reduction to nitrite were fairly similar in the AA and SA groups' genomes, DNRA was more common in the AA genomes and denitrification beyond nitrite in the SA genomes.

Carbon fixation is a necessary step in the anammox bioreactor since the influent media did not contain organic carbon. Only two bacteria in the community could be considered autotrophic primary producers. Brocadia 


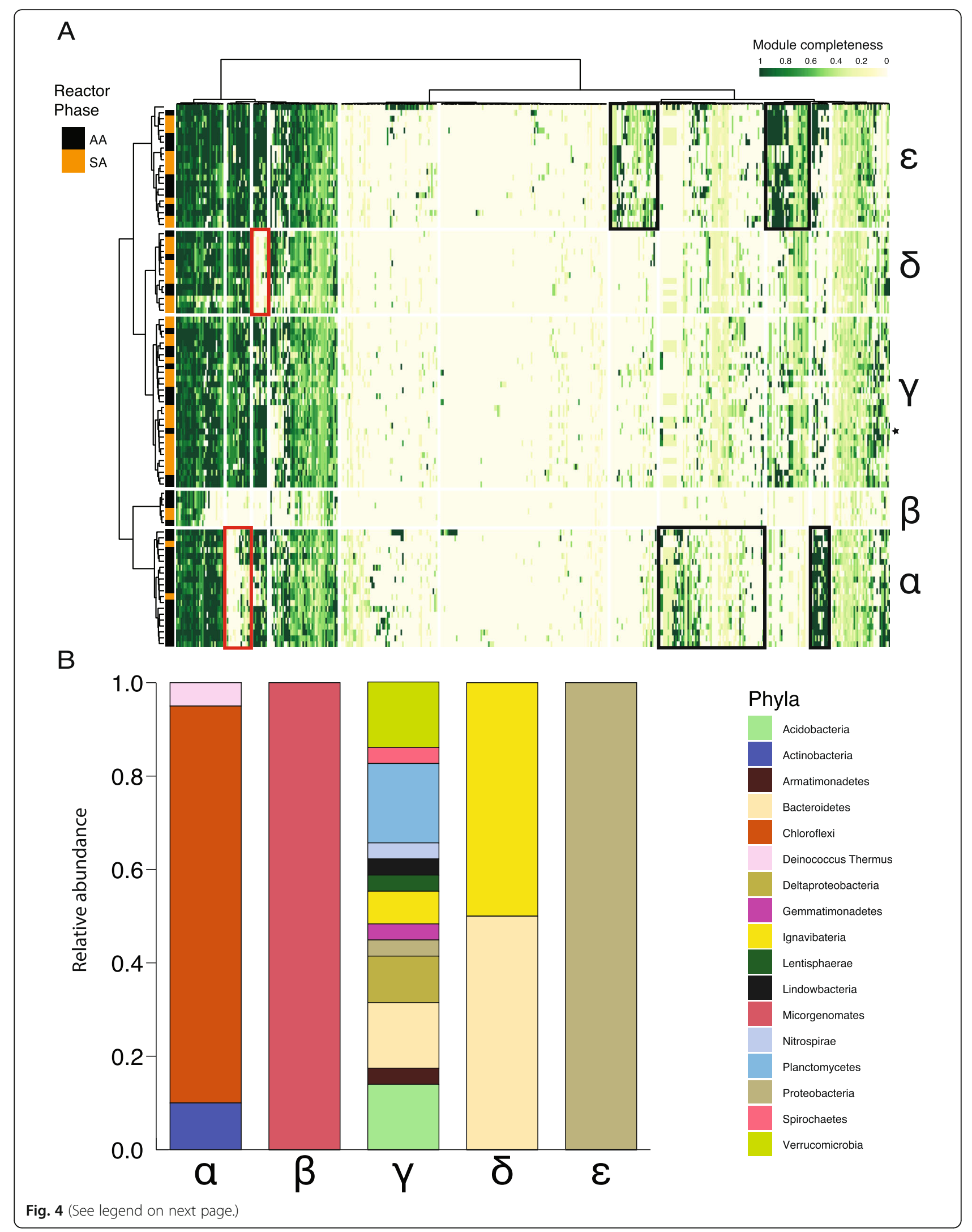


(See figure on previous page.)

Fig. 4 Metabolic profiling of the bacterial community based on KEGG module completeness. a heatmap showing clustering of genomes (rows) by their KEGG module completeness (columns). Completeness ranges from 1 (green) to zero (white). The heatmap is based on a Euclidean distance matrix and clustering with the ward.D method. Genome clustering resulted in 5 clusters (Groups $a-\varepsilon$ ). Rectangles on the heatmap mark module blocks that differentiate the genome groups. Black rectangles on heatmap show module blocks that have increased completeness in a group of bacteria (compared to the others), and red rectangles show decreased completeness. Marks to the left of the heatmap show the division of AA and SA bacteria. A black star to the right of the heatmap marks Brocadia. $\mathbf{b}$ Relative abundance by phyla of members in each metabolic cluster.

was confirmed as a primary producer, fixing carbon via the Wood-Ljungdahl pathway and obtaining energy from the anammox pathway. The second bacterium, LAC_ PROT27 (Proteobacteria, Group AA), might be able to fix carbon by the Calvin cycle and obtain energy from denitrification and could possibly oxidize sulfide to sulfite ( $\operatorname{ds} A B$ is present in the genome). While LAC_ PROT27 was consistently highly abundant in the bioreactor, it was always at least threefolds less abundant than Brocadia (except at time 0). Several other bacteria were also potential autotrophs (or mixotrophs) but were low in relative abundance over the bioreactor's lifespan. Additional information about carbon metabolism and electron transfer can be found in Additional file 1.

\section{Analysis of metabolic selection in the anammox bioreactor}

During the maturation of the anammox bioreactor, the number (Table 1) and diversity (Additional file 1: Table S4) of genomes were both reduced. To examine why certain bacteria were enriched (Group AA) while others were removed (Group SA), we compared the genomes' ability to synthesize metabolites against their ability to acquire nutrients from the environment. For synthesis we checked 24 KEGG modules for amino acids (aa.), 18 modules for vitamins and cofactors, and 28 modules for lipids and fatty acids. For nutrient acquisition we checked 54 KEGG modules for transporters. The mean module completeness across these categories was compared. A complete module implies a bacterium has the functional capability (be it synthesis or transport). Thus, the higher the module completeness of a group, the more likely it is that its members have the relevant functional capability. For statistical analysis, when both data sets (AA and SA) fit a normal distribution, a two sample $T$ test was conducted. When the values did not fit a normal distribution, the ratio between Groups AA and SA was calculated, and used to set a confidence interval (CI, mean \pm $1.64^{*}\left(\mathrm{SD} / \mathrm{n}^{0.5}\right)$, alpha $\left.=0.05\right)$. Values outside of the CI were considered significantly different than the mean.

Synthesis modules for aa. $(p$ value $=0.68)$ and vitamins/cofactors $(p$ value $=0.51$ ) both fit a normal distribution, and no statistically significant differences were found for these categories between the AA and SA bacterial groups. Synthesis modules for lipids and fatty acids did not have a normal distribution, so their ratio was inspected $(\mathrm{CI}$ upper $=1.22$, CI lower $=0.80)$. Six modules were significantly higher and 14 were significantly lower in the AA group vs. the SA group. Group AA had a higher proportion of Gram (+) bacteria, which also added to the difference in module completeness. Transport modules also did not fit a normal distribution. Inspection of the module ratio showed that both the upper CI (2.69) and lower CI (1.74) were higher than a ratio of 1 . Of the 26 transport modules with a ratio $>1.74,18$ were transport systems for organic carbon molecules (sugars, lipids, aa., and cofactors).

These comparisons suggest that a bacterium's ability to acquire nutrients from its environment may be a selective driver in the anammox bioreactor's community. This was particularly highlighted when observing metabolic Group $\alpha$, the dominant metabolic group within the AA bacteria. Members of Group $\alpha$ have a cassette of extracellular proteases and decarboxylases paired with a wide array of transporters (Fig. 5b) that enable acquisition of nutrients from the environment. In addition, the larger ratio of bacteria with auxotrophies in the AA bacteria (Fig. 4a, red rectangles for Groups $\alpha$ and $\delta$ ) hints of a greater reliance on external metabolites from other members of the community.

\section{Metabolic interdependencies between community members}

The bacteria in the AA community have a complex metabolic system, with many bacteria relying on other members to provide them with necessary metabolites. In the mature functioning bioreactor, Brocadia was the only primary producer present. It was also the only bacterium capable of synthesizing vitamin B12. For most other metabolites (e.g., vitamins and cofactors) the possible metabolic interdependencies [31] are less straightforward (Fig. 7 and Additional file 1: Table S5). In the schematic figure, the size of each group reflects its relative abundance in the bioreactor at D437. The arrows point towards the group that potentially receives metabolites it cannot synthesize, and the arrows' sizes reflect the proportion of the examined metabolites that the group needs. Members of Group $\alpha$ (the most dominant group aside from Brocadia) had multiple auxotrophies in the synthesis of vitamins, cofactors, fatty acids, and lipids. Group $\alpha$ could produce a few metabolites needed by other groups. Members of Group $\varepsilon$ were the most 


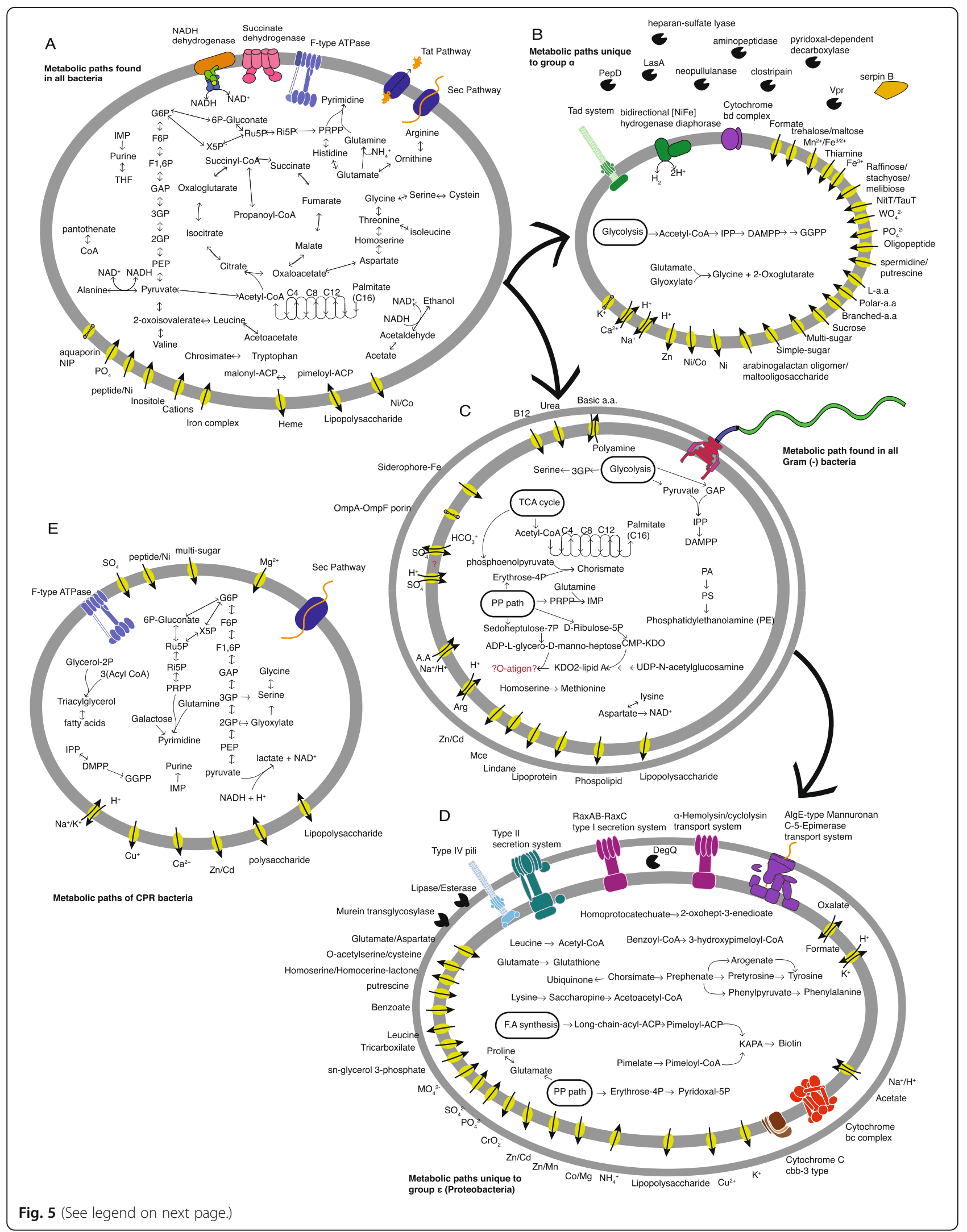


(See figure on previous page.)

Fig. 5 Representative metabolic maps of bacterial groups in the bioreactor. To prevent redundancy, the metabolism is presented in a nested approach with each panel showing only paths unique to the relevant metabolic group. Two exceptions are Group $\beta$ (all detected paths are shown), and Group $\delta$. The latter is not presented here since it shares all paths with Group $\gamma$ and only differs by auxotrophies. (A) Metabolic map of paths that are common to all bacteria in the bioreactor (except Microgenomates and Brocadia sp.). The vast majority of bacteria in the bioreactor are heterotrophs, capable of carbohydrate-based metabolism (glycolysis, pentose phosphate pathway), and amino acid-based metabolism. Some bacteria can respire oxygen and can also ferment (acetate/alanine). (B) Paths unique to Group a. These bacteria have genes for hydrogen oxidation, supporting anaerobic growth, as well as genes for oxidative phosphorylation with cytochrome BD complex. These bacteria have a cassette of extracellular proteases and decarboxylases, paired with a wide array of transporters. They are also potentially capable of synthesizing long chain isoprenoids. (C) Paths found in Gram (-) bacteria (Groups $\gamma, \delta$, and $\varepsilon$ ). Most paths are related to fatty acid and lipid synthesis. Several important precursors (chorismate and IMP) can potentially be synthesized by these bacteria. Motility is also a common feature in these bacteria (via a flagellar motor). (D) Unique paths of Group $\varepsilon$ (Proteobacteria). This group has the potential to synthesize multiple vitamins and cofactors (biotin, pyridoxal, glutathione, etc.), as well as several amino acids (tyrosine, phenylalanine, proline). Another unique feature is the multiple secretion systems present in the bacteria. (E) Metabolic profile of CPR bacteria (Microgenomates). These bacteria are obligate anaerobes that ferment pyruvate. They can only utilize carbohydrates as their carbon source. Some of the bacteria in this group might also be able to synthesize long chain isoprenoids, in the same path as Group a

metabolically diverse and could produce many metabolites needed by other groups. This group could account for most of the auxotrophies of Brocadia. Members of Group $\varepsilon$ could support $75 \%$ of Brocadia's auxotrophies in aa., vitamins, and cofactors, as well as $60 \%$ of its auxotrophies in fatty acids and lipid synthesis (Additional file 1: Table S5). Group $\gamma$ was the smallest group in the bioreactor at D437 and had a mix of auxotrophies and metabolic support potential.

When combining all of the above data, we found that Groups $y$ and $\varepsilon$ both had mutualistic associations with Brocadia (Fig. 7). Group $\varepsilon$ potentially provided more metabolites to Brocadia than it received, whereas Groups a and $\delta$ seemed to gain more from Brocadia than they provided.

\section{Investigating a microbial driven anammox performance destabilization event}

Just before Day 300 of the bioreactor's lifespan, an unexpected anammox performance destabilization event occurred. We hypothesized that some interaction between the anammox bacterium and the co-existing community members led to this event. To evaluate which bacteria might have influenced the destabilization event and which might have been influenced, we relied on two parameters: replication and relative abundance (using coverage). First, we checked for changes in a genome's replication rate (as calculated by iRep [32], see the "Methods" section for detailed explanation) between D166 and D284. Second, we investigated the log-ratio

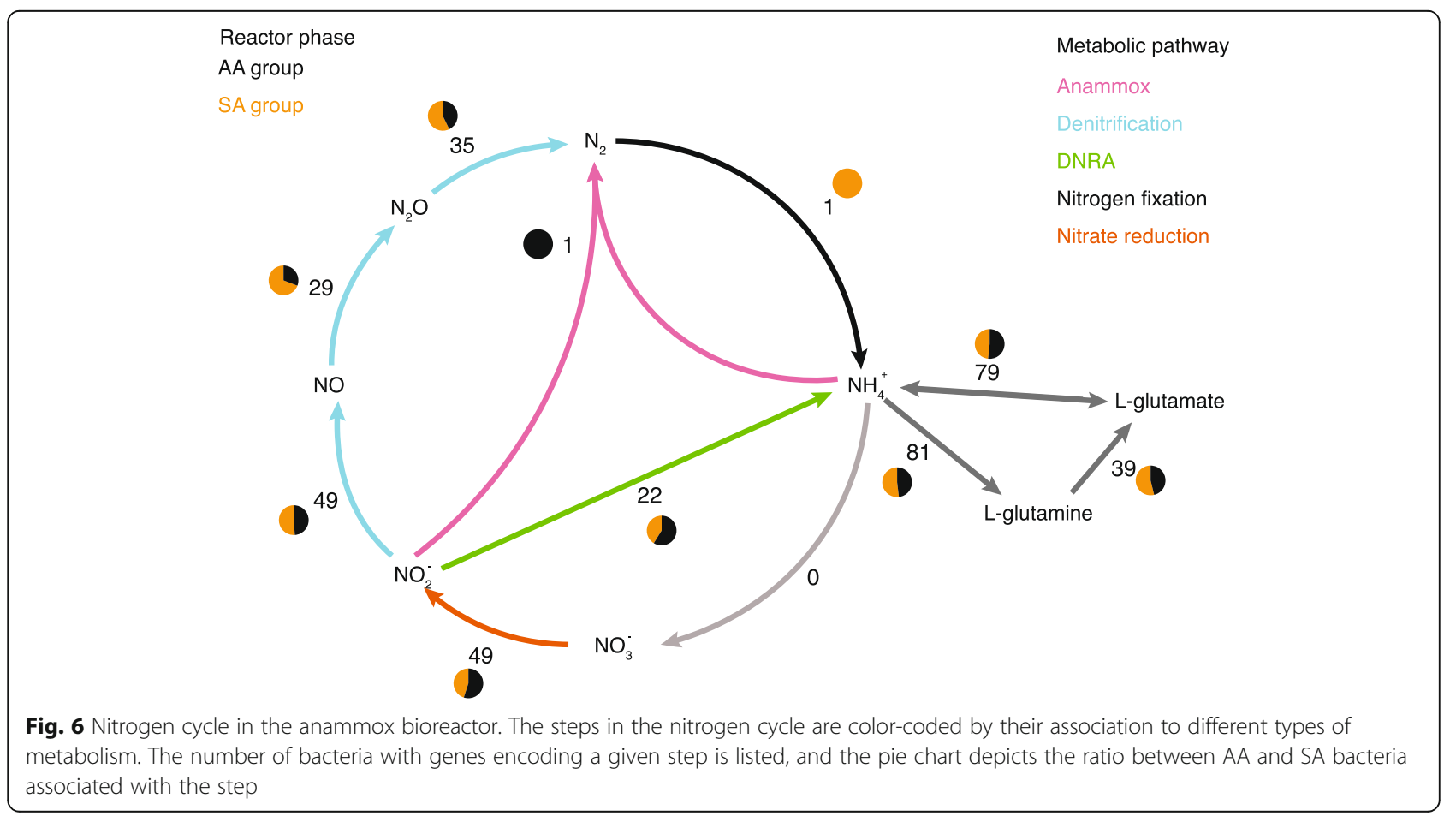


A

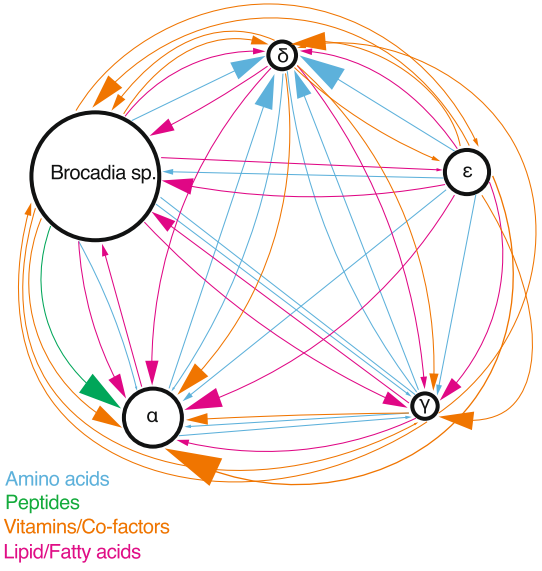

C

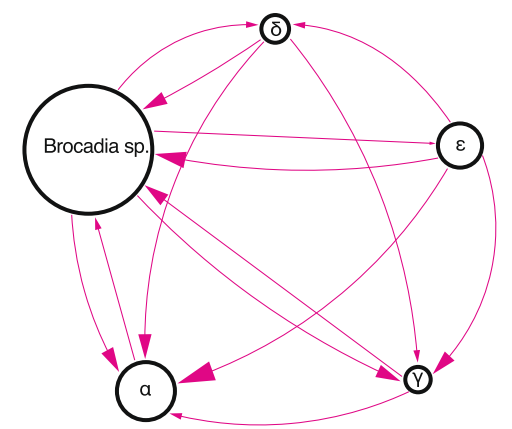

Lipid/Fatty acids
B

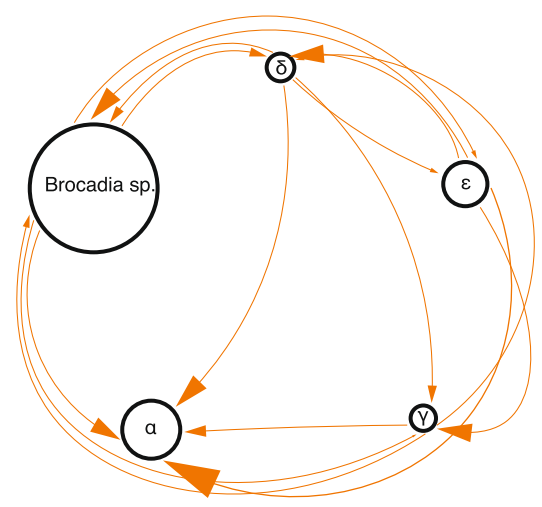

Vitamins/Co-factors

D

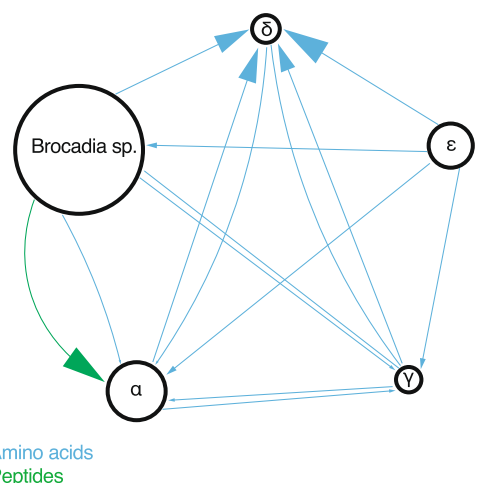

Fig. 7 Potential metabolic interdependencies between the metabolic groups in the anammox bioreactor. a All potential metabolic interdependencies combined. b Metabolic interdependencies for vitamins/cofactors alone. c Metabolic interdependencies for lipids/fatty acids alone. $\mathbf{d}$ Metabolic interdependencies for amino acids and peptides alone. Arrows were assigned according to the absence of a group's ability to synthesize a metabolite, and they connect to all groups that have the ability (there is redundancy in arrows). The arrowhead points at the group that receives the metabolite. The width of the arrow is proportional to the ratio of metabolites of a given type that are provided; amino acids, 20 metabolites; peptides, deduced from proteases and transporters (Fig. 5b); vitamins/cofactors, 10 metabolites; and lipids/fatty acids, 7 metabolites. The size of each group is proportional to their relative abundance at Day 437. Group $\beta$ is not shown since it is assumed that all members obtain all of their nutrients and metabolites from their host. Overall, Groups a and $\delta$ receive the most metabolites and Group $\varepsilon$ receives the least. Group $\delta$ has the highest number of aa. synthesis auxotrophies and can potentially acquire these from many other community members. Group $\varepsilon$ has only a single auxotrophy in vitamin/co-factor synthesis, while most other groups have multiple auxotrophies. Brocadia sp. is the only bacterium capable of vitamin B12 synthesis

(LR) of coverage [33] between D284 and D328. To eliminate bias in microbial loading or sequencing depth, we used four different reference frame genomes (RFg) for the LR calculation [33]. Difference in sequencing depth or microbial load can highly bias comparisons between samples. Higher sequencing depth means higher coverage across the entire sample so it might seem that the abundance of bacteria has increased. To remove that bias, a reference frame genome(s), with little change in relative abundance over time, is chosen. The abundance values of all other genomes within a given time point are divided by the abundance of the RFg before calculating the log-ratio between samples. The internal ratio removes the aforementioned bias between samples. By combining these two parameters, we were able see which bacteria were actively replicating before the performance destabilization event and eliminate biases created by relative abundance results.

Genomes with iRep values for three out of the four time points between D166 and D437 (27, accounting for $>80 \%$ of community) were chosen for this analysis. For each RFg in the LR calculations, significant change was considered for values outside of the confidence interval (CI), calculated for all 127 genomes (Additional file 1: Table S6). A bacterium was considered to influence the destabilization if it both increased in replication prior to the event and had a positive and significantly high LR relative to each RFg. A bacterium was considered to be influenced by the destabilization if it decreased its replication rate prior to the event and its LR was significantly low.

Two Chloroflexi (LAC_CHLX01 and LAC_CHLX10) showed consistent, significant growth across all RFgs 
(Fig. 8a and Table 2), as well as increased replication rates prior to the destabilization event (Fig. 8b). These bacteria likely influenced the destabilization event. Three additional bacteria (the Chloroflexi LAC_CHLX06, LAC_ CHLX09, and Ignavibacteria LAC_IGN05) were also potential influencers, having significant growth based on some of the RFgs. On the other hand, Brocadia (LAC_ PLT02), showed significantly decreased replication rates prior to the destabilization event. Brocadia's replication rate on D284 (1.07) indicates that only 7\% of the bacterium's population was actively replicating. At other time points, Brocadia's replication rate climbed as high as 2.13 , indicating that $100 \%$ of the bacterium's population was actively replicating. One additional bacterium (LAC_ PROT22, a Proteobacteria from Group AA) showed decreased replication and growth (under two RFgs).

Next, we next investigated the nitrogen metabolism of the 27 bacteria, specifically the three metabolic paths that compete for nitrite, i.e., anammox, denitrification, and DNRA (Fig. 8c). Denitrification was subdivided into three steps $\left(\mathrm{NO}_{2}\right.$ reduction, $\mathrm{NO}$ reduction, and $\mathrm{N}_{2} \mathrm{O}$ reduction); DNRA is a single-step process. Anammox could only be performed by Brocadia. Only a single bacterium (LAC_BAC20) could perform full denitrification. All bacteria capable of DNRA were also capable of partial denitrification. When allowing for a bacterium to
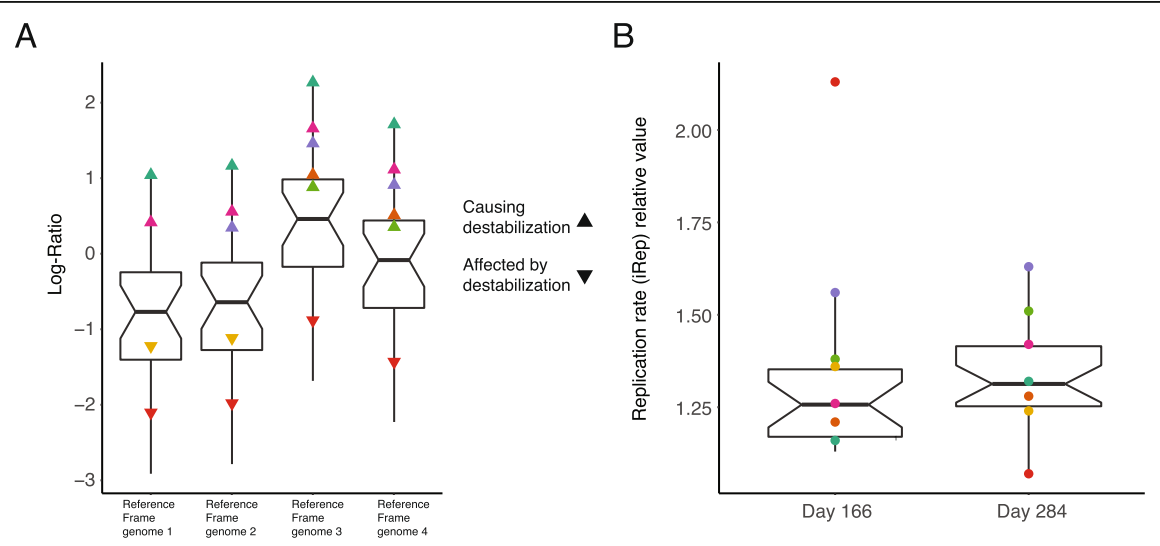

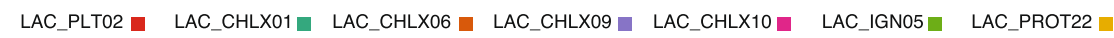

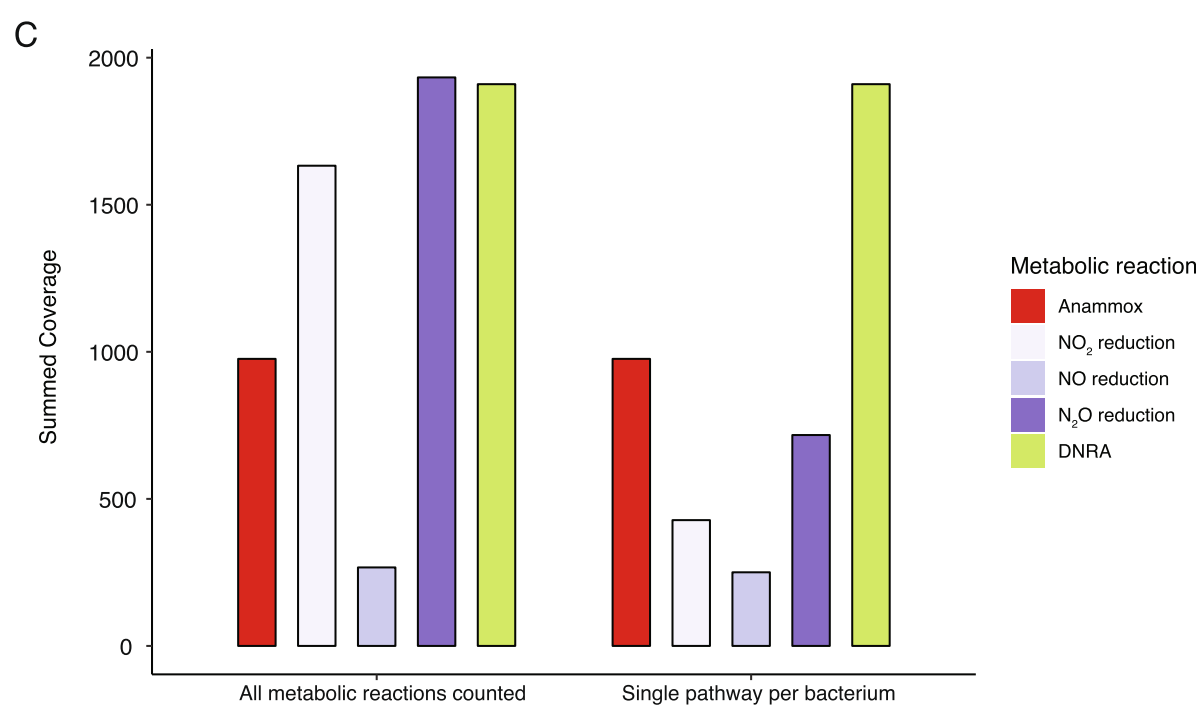

Fig. 8 The tracking of bacterial growth and nitrogen metabolism in relation to the anammox performance destabilization. a Distribution of Logratio changes for selected bacteria between D328 and D284, using different genomes as reference frames. Bacteria that are significantly affected or considered to affect the destabilization event are color-coded. Down-pointing triangles depict bacteria negatively affected by the destabilization and upwards-pointing triangles indicate bacteria that likely caused (or contributed) to the destabilization. $\mathbf{b}$ Replication rate values on Days 166 and 284. Bacteria are color-coded the same as in panel a. c Relative abundance of nitrogen metabolism pathways in the selected bacteria. Denitrification is divided into its reaction steps. Anammox is considered a single path since only a single bacterium can perform it (Brocadia-LAC_PLT02). DNRA from NO2 is a single-step reaction. Abundance was calculated twice, once allowing multiple pathways to occur within each bacterium, and once after choosing a single pathway per bacterium (based on potential energy gain). 
Table 2 Log-ratio and replication rates (iRep) for select bacteria

\begin{tabular}{lllllll}
\hline Genome & $L_{(R F 1)}$ & $L_{(R F 2)}$ & $L R_{(R F 3)}$ & $L R_{(R F 4)}$ & iRep D166 & iRep D284 \\
\hline LAC_CHLX01 & 1.05 & 1.17 & 2.27 & 1.73 & 1.16 & 1.32 \\
LAC_PROT02 & -1.54 & -1.41 & -0.31 & -0.85 & 1.17 & 1.30 \\
LAC_PROT03 & -1.50 & -1.37 & -0.27 & -0.81 & 1.32 & 1.40 \\
LAC_CHLX02 & -2.91 & -2.79 & -1.68 & -2.23 & 1.16 & 1.17 \\
LAC_ACD03 & -0.99 & -0.86 & 0.24 & -0.30 & 1.19 & 1.35 \\
LAC_BACT04 & -0.77 & -0.64 & 0.46 & -0.08 & 1.26 & 1.26 \\
LAC_IGN05 & -0.33 & -0.20 & 0.90 & 0.36 & 1.38 & 1.51 \\
LAC_PROT19 & -2.09 & -1.96 & -0.86 & -1.40 & 1.22 & 1.32 \\
LAC_IGN07 & -0.98 & -0.85 & 0.25 & -0.29 & 1.30 & 1.48 \\
LAC_PROT21 & -1.18 & -1.06 & 0.04 & -0.50 & $\mathrm{NA}$ & 1.29 \\
LAC_BAC19 & -0.13 & 0.00 & 1.10 & 0.56 & $\mathrm{NA}$ & 1.38 \\
LAC_BAC20 & -1.17 & -1.04 & 0.06 & -0.48 & 1.13 & 1.19 \\
LAC_BACT12 & -1.72 & -1.59 & -0.49 & -1.03 & 1.14 & 1.18 \\
LAC_NIT01 & -1.93 & -1.80 & -0.70 & -1.24 & 1.25 & 1.31 \\
LAC_CHLX06 & -0.18 & -0.05 & 1.05 & 0.51 & 1.21 & 1.28 \\
LAC_PROT22 & -1.23 & -1.10 & 0.00 & -0.54 & 1.36 & 1.24 \\
LAC_PLT02 & -2.12 & -1.99 & -0.89 & -1.43 & 2.13 & 1.07 \\
LAC_CHLX07 & 0.00 & 0.13 & 1.23 & 0.69 & 1.69 & NA \\
LAC_BAC21 & -1.31 & -1.18 & -0.08 & -0.62 & 1.13 & 1.25 \\
LAC_CHLX09 & 0.25 & 0.37 & 1.48 & 0.93 & 1.56 & 1.63 \\
LAC_CHLX10 & 0.43 & 0.55 & 1.66 & 1.11 & 1.26 & 1.42 \\
LAC_BAC22 & 0.27 & 0.40 & 1.50 & 0.96 & NA & 1.39 \\
LAC_BAC23 & -0.31 & -0.18 & 0.92 & 0.38 & 1.17 & 1.17 \\
LAC_PROT27 & -0.35 & -0.23 & 0.88 & 0.33 & 2.06 & 1.56 \\
LAC_PROT28 & -0.69 & -0.56 & 0.54 & 0.00 & 1.33 & 1.27 \\
LAC_PROT30 & -0.48 & -0.36 & 0.75 & 0.20 & NA & 1.89 \\
LAC_D-T02 & -0.43 & -0.30 & 0.80 & 0.25 & NA & 1.59 \\
\hline
\end{tabular}

have multiple pathways (Fig. 8c), $\mathrm{NO}_{2}$ reduction via DNRA (nrfAH), and denitrification (nirS/nirK) appeared to be equally dominant during the anammox performance destabilization event (D328). While $\mathrm{N}_{2} \mathrm{O}$ reduction was also dominant, a bottleneck appeared to exist at the second step, NO reduction. When we assume that the bacteria capable of both DNRA and partial denitrification choose the path that yields the most energy [34], we can remove the partial denitrification pathway (Fig. 8c). Under this assumption, DNRA was clearly revealed as the dominant process occurring in the bioreactor during the anammox performance destabilization event. All bacteria that were shown to affect the destabilization event were DNRA bacteria. In addition, the destabilizing bacteria belong to metabolic Groups a (LAC_CHLX01, LAC_CHLX06, LAC_ CHLX09, and LAC_CHLX10) and $\delta$ (LAC_IGN05). Both of these groups were reliant on other members for organic carbon (Figs. 5b and 7).

\section{Core anammox community}

Resulting genomes from our study, in combination with genomes from two previous anammox metagenomic studies, Speth et al. [18] (22 genomes) and Lawson et al. [20] (15 genomes), provide strong evidence to support a core anammox community (Fig. 9). The relative abundances of bacteria from the dominant phyla across these three bioreactors were fairly similar: in each bioreactor, the anammox, along with Chloroflexi, Ignavibacteria, and Proteobacteria bacteria, composed $>70 \%$ of the community (Fig. 9b).

Due to the significantly larger genome yield and time series analysis in this study, our bioreactor shared more genomes with each of the other bioreactors than the other bioreactors shared between themselves. In total, 21 genomes from our bioreactor were closely related to those from at least one of the two other bioreactors, 17 of which were present at the last time point, D437 (Additional file 1: Table S7). The related bacteria accounted for $50 \%$ and $93 \%$ of the Speth et al. and Lawson et al. genomes, respectively. The bioreactor studied by Speth et al. was different from the other two bioreactors because it was amended with oxygen to perform partial nitritation and anammox within the same bioreactor, while the others performed anammox only.

A more focused phylogenetic tree of Planctomycetes shows that the Brocadia in our bioreactor and in the Lawson et al. bioreactor are the same species (Brocadia sapporensis [35]), while the Brocadia species from the Speth et al. bioreactor is different (Brocadia sinica) (Additional file 1: Figure S3).

\section{Discussion}

In this study we present an in-depth analysis of the development of an anammox community from seed to stable state (through several perturbations) in an anaerobic membrane bioreactor. By combining several methodologies, we are able to gain important insights into the dynamics and interactions of more than 100 species in the bioreactor community [36, 37].

The first perturbation of the bioreactor, a mechanical malfunction combined with inoculum amendments, changed the trajectory of the community succession. This can be seen from the relative abundance-based grouping (Figs. 2 and 3) and the detected strain shifts. The first inoculum amendment had a much stronger effect on community assembly than the subsequent bioreactor malfunction and second amendment $[38,39]$. The large shift in the community occurred between Days 96 and 152, after which the community trajectory became fairly consistent until Day 290. The oscillations after Days 350 are likely due to differences in sequencing depths. Large changes in community structure due to inoculations are a real concern for large-scale bioreactors, where the influent contains constantly changing communities of bacteria [40, 41]. It is unclear if members of Group C, 


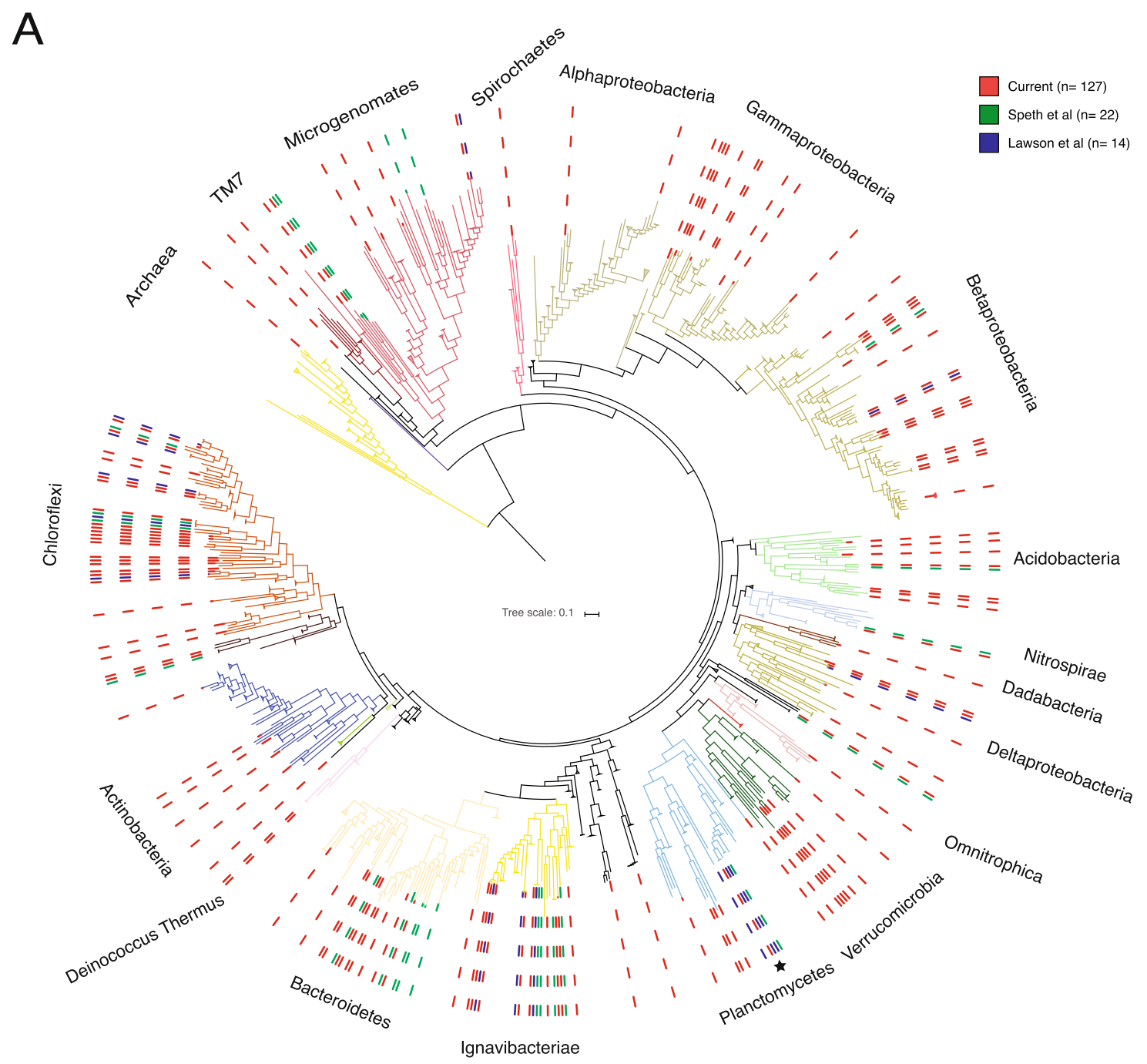

B

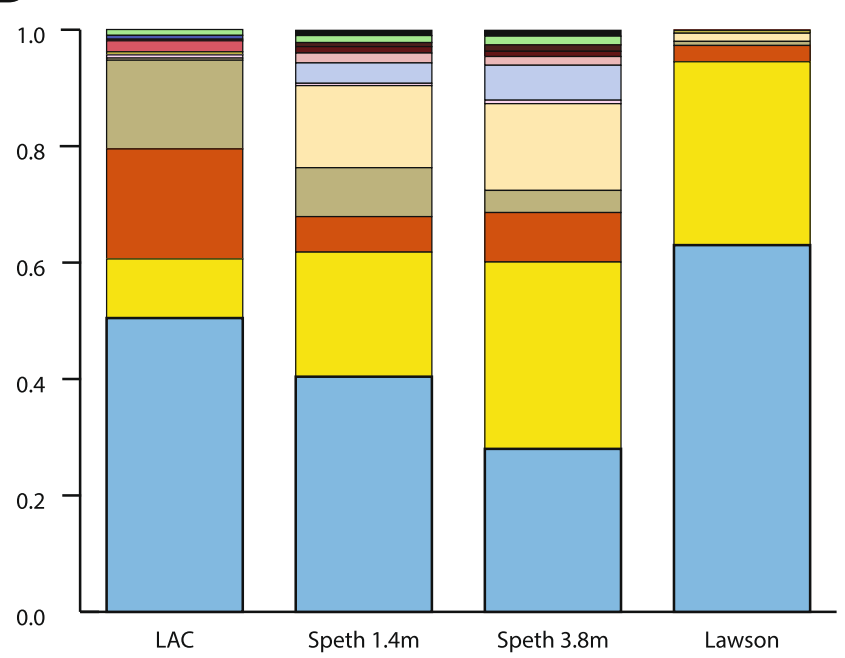

$\square$ Acidobacteria

- Actinobacteria

- Armatimonadetes

$\square$ Bacteroidetes

$\square$ Chloroflexi

$\square$ Deinococcus Thermus

$\square$ Deltaproteobacteria

$\square$ Ignavibacteria

$\square$ Microgenomates

$\square$ Nitrospirae

$\square$ Omnitophica

- Parcubacteria

$\square$ Planctomycetes

$\square$ Proteobacteria

Fig. 9 (See legend on next page.) 
(See figure on previous page.)

Fig. 9 Phylogenetic analysis of three anammox microbial communities. a A maximum likelihood tree based on the alignment of 15 concatenated ribosomal proteins. In the construction of the tree 3225 reference sequences were used, with genomes from current and previous genomecentric studies on anammox communities. Genomes from the current anammox community are marked with a red dashed line, genomes from two previously studied communities, Speth et al. and Lawson et al., are marked with green and blue dashed lines, respectively. Nodes containing only reference genomes were collapsed for ease of view. Collapsed nodes are depicted as triangles and their size is relative to the number of bacteria they contain. The black star marks Brocadia. b Relative abundance of major phyla in the three microbial communities. Current community reference data was calculated from Day 437 only. The relative abundance of Brocadia sp. comprises nearly all of the relative abundance attributed to phylum Planctomycetes (with small contributions from other members of the phylum). The most abundant phyla (Chloroflexi, Ignavibacteria, and Proteobacteria) consistently account for $>70 \%$ of the communities. The phyla colors follow the ggkbase color scheme and the major phyla are shown in the legend

which were dominant in the nascent anammox community, would have better supported the bioreactor's anammox performance [38]. Looking at their metabolism may offer a few clues: of the 14 genomes clustered into the nascent anammox community, six were of Group $\varepsilon$ and four were of Group $\gamma$, both of which were considered to maintain a mutualistic or commensal relationship with the anammox bacterium. In addition, denitrification was more common than DNRA in Group C. Two genomes (LAC_ACD03 and LAC_ PROT30) from the nascent anammox community were included in the focused investigation of the anammox performance destabilization. Both had no significant effect on the event nor were they significantly affected by it.

The metabolic analysis of the matured community showed that transport systems for nutrients (mainly organic carbon) were the most enriched in the community. The ability of bacteria to utilize available nutrients in the environment [42] has been shown before and has been proposed to explain dominance in nutrient-rich environments [43]. However, when such bacteria favor acquisition over synthesis, it can further stress a community that is reliant on a slow growing primary producer for nutrients [44-47]. Members of Groups $\alpha$ and $\delta$ have these characteristics, and some are implicated in the anammox performance destabilization event.

The aforementioned destabilization event took place after nearly 100 days of high performance, and no external factors could explain the sudden destabilization and performance crash. By combining information about replication rates and changes in relative abundance of community members, we were able to identify several bacteria that likely affected the performance crash. Analysis of composite data (such as relative abundance) for true changes in community structure has many pitfalls [33]. In addition, the response of a bacterium to an event, in this case an increase in relative abundance following the performance destabilization, cannot be deduced to mean it had any effect on the event. We were fortunate to have taken a metagenomic sample the week prior to the destabilization event. By measuring replication rate changes as well as relative abundance changes, we could better deduce a possible causative effect. A bacterium that increases its replication rates prior to the event and increases in relative abundance due to the event is more likely to have a causative effect. While most bacteria had higher replication rates at D284 compared to D166 (17 of 22 bacteria with values in both days), only five bacteria significantly increased in relative abundance following the event. Genes conferring DNRA and partial denitrification capabilities were detected in these bacteria. These types of bacteria could improve bioreactor performance if they remove nitrate and excess nitrite, but they could be detrimental if they compete with anammox for nitrite or allow for the buildup of nitrite. Here, the equilibrium between support of the anammox process and disruption of the anammox process was tilted towards the latter.

Two possible scenarios for nitrogen metabolism are consistent with the bioreactor's performance that exhibited decreased nitrogen removal and increased ammonium in the effluent leading up to the destabilization event. One scenario is described by DNRA dominance and the second by nitrite reduction to nitric oxide and its subsequent leakage from the system. The first scenario provides more energy to the bacteria [34], so we speculate it is more likely. Brocadia has genes required for DNRA, but given the high nitrogen removal rate by the anammox process leading up the disturbance; it can be assumed that Brocadia would not be primed to carry out the DNRA reaction on such a short timescale. However, DNRA could potentially be used by Brocadia for detoxification by cycling potentially toxic excess nitrite back to ammonium, where it could then participate in the anammox reactions $[18,20]$.

The replication rate of Brocadia prior to the performance destabilization was down to 1.07 , from 2.13 on D166. A replication rate of 1.07 is equivalent to only $7 \%$ of the population actively replicating, which can explain the large decrease in relative abundance in the following time point. This also hints that the process leading to the destabilization event occurred prior to D284. Whether the process could be assigned to a single specific occurrence, or if it is an additive process culminating in a break from equilibrium among members of the community, is a gap that future research should address. 
A broader investigation of metabolic interdependencies within the community sheds light on the stability of the anammox community. Brocadia is the source of organic material in the community but obtains essential metabolites from community members, especially Proteobacteria. This forms a basis for a mutual symbiotic relationship. On the other hand, Chloroflexi, comprising the largest group of bacteria besides Brocadia, receive numerous metabolites while apparently providing few in return. They are characterized by an array of extracellular proteases and amylases, likely used to breakdown the extracellular matrices formed by Brocadia. Chloroflexi, as a group, are most associated with anammox bacteria and form a large fraction of the core community. They also account for the majority of the destabilizing bacteria. Together, the results point to a parasitic symbiosis. While anammox bacteria generate sufficient organic carbon to support the growth of its co-occurring heterotrophic microorganisms, the tipping point between stable and unstable operation and the factors that control it have not been fully identified. Input changes may be able to restore anammox activity, but this is only an empirical solution. In full-scale anammox bioreactors where influent organic carbon is essentially ubiquitous, heterotrophic dominance could persist without some sort of active countermeasure. Therefore, future research should target the inhibition of potential destabilizing heterotrophs.

Previous studies have discussed a potential core anammox community [12-16]. With the exception of very few studies, all such work has been conducted with single-gene markers. Our analysis of an anammox community is the largest to date and thus expands the ability to test this hypothesis. Our results support the existence of a core community, while identifying factors that differentiate communities. The high similarity among bacterial communities originating from three distinct anammox bioreactors $[18,20]$ strongly suggests a global core anammox microbial community. In the construction of the phylogenetic tree, we used $>3000$ reference genomes originating from diverse environments. Through this analysis, we found that the anammox community forms distinct clades at the species level, despite the sheer number and diversity of sources. More than half of the bacteria did not have species level relatives, and an additional $26 \%$ only had a relative found in our anammox bioreactor or in a previous anammox study $[18,20]$. Together, nearly $80 \%$ of the bacteria are unique to anammox bioreactors, so it is clear that the anammox bioreactor selects for a unique set of bacteria. Parameters that increased the differences between communities are the species of the anammox bacterium and the bioreactor configuration. Since both parameters relate to the same bioreactor [18], we cannot conclude which has a stronger effect.

\section{Conclusions}

Here we present the largest-to-date metagenomic analysis of a microbial community in an anammox bioreactor. Our results support the growing body of literature that suggests that anammox communities are unique and may share a core microbial community. We identified a distinct phylogenetic profile across reported metagenomic analyses of anammox bioreactors. In subsequent analyses of our metagenomes, we identified metabolic traits associated with the core microbial community that are distinguishable from other bacteria present in the source sludge inoculum. In addition, our time series analysis included a biologically driven period of anammox performance destabilization. We identified an increase in replication rates for several bacteria just prior to the event. Further analysis revealed that these bacteria contain genes conferring DNRA, which puts them in direct competition with the Brocadia sp. for nitrogen resources. Combined, our results provide a possible mechanistic explanation for the performance shift of the anammox bioreactor and advance the comprehensive control of this promising technology. However, further work is needed to elucidate the precise mechanisms that govern anammox community interactions and to predict performance destabilization events.

\section{Methods}

\section{Bioreactor operation}

A laboratory-scale, anammox anaerobic membrane bioreactor (MBR) with a working volume of $1 \mathrm{~L}$ was constructed and operated for over 440 days (Additional file 1: Figure S4). The bioreactor was originally inoculated with approximately $2 \mathrm{~g}$ volatile suspended solids (VSS) $\mathrm{L}^{-1}$ of biomass from a pilot-scale deammonification process treating sidestream effluent at San Francisco Public Utilities Commission (SFPUC) in San Francisco, $\mathrm{CA}$. The bioreactor was re-inoculated with similar concentrations of biomass from the same source on Days 147 and 203. Synthetic media containing ammonium, nitrite, bicarbonate, and trace nutrients (meant to mimic sidestream effluent at a municipal wastewater treatment plant) was fed to the bioreactor (Additional file 1: Table S8). For the first 154 days of operation, the bioreactor was kept under nitrite-limiting conditions to prevent inhibitory conditions due to the buildup of nitrite, and influent ammonium and nitrite concentrations ranged from 200 to $300 \mathrm{mg} \mathrm{N} \mathrm{L}^{-1}$ and 100 to $300 \mathrm{mg} \mathrm{N} \mathrm{L}^{-1}$, respectively. On Day 154, ammonium and nitrite concentrations were adjusted to the theoretical anammox stoichiometric ratio, 1:1.32. Afterwards, influent ammonium and nitrite concentrations were maintained at this ratio. Ammonium ranged from 200 to $500 \mathrm{mg} \mathrm{N} \mathrm{L}^{-1}$ and nitrite 265 to $660 \mathrm{mg} \mathrm{N} \mathrm{L}^{-1}$. On Day 353, influent 
concentrations of copper, iron, molybdenum, and zinc were increased based on literature suggestions [22-25].

The bioreactor was operated in a continuous flow mode. For the first 145 days, the hydraulic retention time (HRT) was maintained at $48 \mathrm{~h}$; afterwards it was reduced to $12 \mathrm{~h}$. No solids were removed from the bioreactor for the first 100 days of operation; afterwards, the solids retention time (SRT) was reduced to 50 days. A polyvinylidene fluoride hollow fiber membrane module with a 0.4$\mu \mathrm{m}$ pore size and total surface area of $260 \mathrm{~cm}^{2}$ (Litree Company, China) was mounted in the bioreactor. Temperature was maintained at $37^{\circ} \mathrm{C}$ with an electric heating blanket (Eppendorf, Hauppauge, NY). Mixing was provided by an impeller at a rate of $200 \mathrm{rpm}$. Mixed gas was supplied continuously to the bioreactor $\left(\mathrm{Ar}: \mathrm{CO}_{2}\right.$ $=95: 5 ; 50 \mathrm{~mL} \mathrm{~min}^{-1}$ ) to eliminate dissolved oxygen and maintain a circumneutral $\mathrm{pH}$ range of 6.9-7.2. Influent and effluent concentrations of ammonium, nitrite, and nitrate were measured approximately every other day using $\mathrm{HACH}$ test kits ( $\mathrm{HACH}$, Loveland, $\mathrm{CO}$ ), as described in the manufacturer's methods 10031, 10019, and 10020, respectively.

\section{Biomass collection and DNA extraction}

Biomass samples were extracted via syringe from the bioreactor every $2-10$ days, flash frozen in liquid nitrogen, and stored frozen at $-80{ }^{\circ} \mathrm{C}$ until use. Genomic DNA was extracted from the samples using the DNeasy PowerSoil Kit (Qiagen, Carlsbad, CA), as described in the manufacturer's protocol. Extracted DNA was quantified with a NanoDrop Spectrophotometer (Thermo Scientific, Waltham, MA), and normalized to approximately $10 \mathrm{ng} / \mu \mathrm{L}$ with nuclease-free water (Thermo Scientific, Waltham, MA). All genomic DNA samples were stored at $-20^{\circ} \mathrm{C}$ until use. For shotgun metagenomic sequencing, samples were sent to the Joint Genome Institute (JGI) in Walnut Creek, CA. There, DNA quality was assessed prior to library preparation and sequencing (150 bp pair-end) on the Illumina HiSeq $25001 \mathrm{~T}$ sequencer (Illumina, San Diego, CA). For 16S rRNA sequencing, samples were sent to the Institute for Environmental Genomics at the University of Oklahoma. There, DNA quality was assessed prior to library preparation and amplicon sequencing on the Illumina MiSeq sequencer (Illumina, San Diego, CA).

\section{Metagenomic sequencing, assembly, and binning}

Resulting sequences from each time point were processed separately, following the ggKbase SOP (https://ggkbase-help. berkeley.edu/overview/data-preparation-metagenome/).

Briefly, Illumina adapters and trace contaminants were removed (BBTools, GII) and raw sequences were qualitytrimmed with Sickle [48]. Paired-end reads were assembled using IDBA_UD with the pre-correction option and default settings [49]. For coverage calculations, reads were mapped with bowtie2 [50]. Genes were predicted by Prodigal [51] and predicted protein sequences were annotated using usearch [52] against KEGG, UniRef100, and UniProt databases. The $16 \mathrm{~S}$ rRNA gene and tRNA prediction was done with an inhouse script and tRNAscanSE [53], respectively. At this point, the processed data was uploaded to ggKbase for binning.

Manual binning was performed using the ggKbase tool. The binning parameters were $\mathrm{GC} \%$ and coverage (CV) distribution, and phylogeny of the scaffolds. Quality of the manual bins was assessed by the number of Bacterial Single Copy Genes (BSCG) and Ribosomal Proteins (RP) found in each bin (aiming at finding the full set of genes, while minimizing multiple copies). In addition to manual binning, automated binning was done using four binners: ABAWACA1 [29], ABAWACA2, CONCOCT [54], and Maxbin2 [55]. For all, the default parameters were chosen.

All bins from both automatic and manual binning tools were input into DASTool [56] to iterate through bins from all binning tools and choose the optimal set of bins. checkM was run to analyze genome completeness [26]. The scaffold-to-bin file created by DASTool was uploaded back to ggKbase and all scaffolds were rebinned to match the DASTool output. Each of the new bins was manually inspected and scaffolds suspected of being falsely binned were removed.

After inspecting the first round bins, we improved the high coverage bins by subsampling the read files, and repeating the SOP above [57]. In addition, refinement of the Brocadia Genome bins was done with ESOMs [58] (Additional file 1: Supplemental methods).

\section{Post binning analysis}

Unique representative genomes were determined by the dereplication tool, dRep [59], using a 95\% threshold for species level clustering. Within each cluster, the representative genome was chosen based on their completeness, length, N50, contamination, and strain heterogeneity. In several clusters with higher heterogeneity, a second strain was chosen (Additional file 1: Table S2). The strain threshold was set at $2 \%$ difference.

All representative and strain genomes were curated by correcting scaffolding errors introduced by idba_ud, using the ra2.py program [29]. Following curation, the genomes were processed again for gene calling and annotation (see above for details). The replication rates of bacteria can be inferred from examining the coverage ratio between the origin of replication and the terminus of replication. In a population that is not replicating there will be no difference in the coverage so the ratio would be one. If the population is replicating, we expect the ratio to be $>1$ since there would be replication forks that have not finished replicating and thus the coverage 
towards the origin of replication would be higher than that of the terminus. Calculating replication rate is more complicated in metagenomic samples but it is still possible to look at overall trends in coverage across the genome. Analysis of replication rates at different time points was performed with the iRep program [32] using the default parameters (Additional file 1: Table S9). Briefly, iRep calculates replication rate by measuring sequencing coverage trend that results from bi-directional genome replication from a single origin of replication. The program uses only high-quality draft genomes $(\geq 75 \%$ complete, $\leq 175$ fragments/Mbp sequence, and $\leq 2 \%$ contamination). Since iRep is a measure of a trend it does not have any units.

Raw reads were submitted to the National Center for Biotechnology Information (NCBI) Genbank, under project accession number PRJNA511011. In addition, the representative and strain genomes were uploaded to ggkbase as two separate projects (https://ggkbase.berkeley. edu/LAC_reactor_startup/organisms and https://ggkbase. berkeley.edu/LAC_reactor_strains/organisms).

\section{Phylogenetic analysis and core anammox analysis}

The taxonomic affiliation of each genome was initially assigned in ggKbase, based on the taxonomic annotation of genes in the scaffolds. For each hierarchical taxonomic level, the taxonomy was decided if at least $50 \%$ of genes had a known taxonomic identification.

Phylogenetic analysis of the genomes (current study, Speth et al. [18], and Lawson et al. [20]) was based on a set of 15 ribosomal proteins [60]. Each gene was aligned separately to a set of 3225 reference genomes, followed by concatenation while keeping the aligned length of each gene intact. A preliminary tree was created by adding the queried genomes to the reference tree using pplacer v1.1.alpha19 [61] and a set of in-house scripts. The tree was uploaded to iTOL [62] for visualization and editing. After initial inspection, we decided to reduce the tree in preparation for creating a maximum likelihood tree. Large phyla with no representatives in an anammox sample were removed (approximately 1000 sequences). The remaining sequences were aligned by MUSCLE [63] and a RAxML tree built in The CIPRES Science Gateway V. 3.3 [63, 64].

For the analysis of phylogenetic distance between different anammox community members, we used the APE package [65] in $R[66,67]$ to extract the distance matrix. Species level distance was set at $5 \%$ of the longest measured distance on the tree. The $\mathrm{R}$ script and RData files for the analysis of related species, community dynamics, and metabolic capacities were uploaded to figshare (https://figshare.com/projects/ Anammox_Bioreactor/59324).

\section{S rRNA gene sequencing, processing, and analysis}

DNA samples, taken at 55 time points across the lifespan of the bioreactor, were sent to the Institute for Environmental Genomics at the University of Oklahoma (Norman, OK) for amplification of the variable 4 (V4) region of the $16 \mathrm{~S}$ rRNA gene, library preparation, and amplicon sequencing. The full protocol was previously described in Wu et al. [68]. In summary, the V4 region of the bacterial $16 \mathrm{~S}$ rRNA gene was amplified from DNA samples using primers 515F (5'-GTGCCAGCMGCCGCGG-3') and 806R (3'-TAATCTWTGGVHCATCAG-5'), with barcodes attached to the reverse primer. Amplicons were pooled at equal molarity and purified with the QIAquick Gel Extraction Kit (QIAGEN Sciences, Germantown, $\mathrm{MD})$. Paired-end sequencing was then performed on the barcoded, purified amplicons with the Illumina MiSeq sequencer (Illumina, San Diego, CA).

Subsequent sequence processing and data analysis were performed in-house using MOTHUR v.1.39.5, following the MiSeq SOP [69, 70]. In summary, sequences were demultiplexed, merged, trimmed, and quality filtered. Unique sequences were aligned against the SILVA $16 \mathrm{~S}$ rRNA gene reference alignment database [71]. Sequences that did not align to the position of the forward primer were discarded. Chimeras were detected and removed. The remaining sequences were clustered into operational taxonomic units (OTUs) within a 97\% similarity threshold using the Phylip-formatted distance matrix. Representative sequences from each OTU were assigned taxonomic identities from the SILVA gene reference alignment database [71]. Sequences that were not classified as bacteria were removed. Remaining OTUs were counted, and the 137 most abundant OTUs (accounting for up to $99 \%$ of sequence reads within individual samples) were transferred to Microsoft Excel (Microsoft Office Professional Plus 2016) for downstream interpretation and visualization. The read files from all time points, as well as the 137 most abundant OTUs were uploaded to figshare (https://figshare.com/projects/Anammox_Bioreactor/59324).

In order to correlate genome-based OTUs to $16 \mathrm{~S}$ rRNA gene-based OTUs, 16S rRNA gene sequences were extracted from the representative genomes and combined with the representative sequences from the 137 most abundant $16 \mathrm{~S}$ rRNA gene-based OTUs. If a representative genome did not contain the V4 region of the 16S rRNA gene, the region was pulled from another genome in the same cluster. The combined 16S rRNA gene sequences were aligned following the protocol described above, and those sharing at least $99 \%$ average nucleotide identity were assumed to represent the same microorganism [72].

\section{Community dynamics analysis}

The paired sequence reads from all time points were mapped to the set of reference genomes using bowtie2 [50], 
followed by calculations for coverage (average number of reads mapped per nucleotide) and breadth (percent of genome covered by at least one read) for each genome per time point [73]. The multiplication of the two values was then used to calculate the estimated relative abundance. These steps were done to negate the biases created by repetitive sequences that occur more often in partial genome bins.

Association between genomes was tested by calculating pairwise correlation for all genomes by relative abundance. Rho values (ranging from -1 to 1 ) were used to create a distance table (Euclidean distance), followed by clustering with the ward.D method. The resulting clusters were marked A-D. To test for the association of genomes and clusters to time points, we ran a nMDS analysis (non-parametric MultiDimensional Scaling) with genomes and time points. Each genome was colored by its relative abundance cluster on the 2D projection of the nMDS.

For relative abundance changes, the estimated relative abundances of genomes were divided by the sum of all estimated relative abundance values per time point. For a clearer resolution of changes in the four relative abundance groups, the Brocadia (part of Group D) was presented separately.

\section{Metabolic analysis}

The functional profiles of the genomes were evaluated using KEGG KAAS [74], with Hidden Markov Models for shared KEGG Orthologies (KOs) [27, 28]. From this, we received the KEGG annotation (KO number) for all open reading frames and a completeness value for each KEGG module. $\mathrm{KO}$ annotations that were questionable were removed from the analysis.

From the KO list, we created a presence-absence matrix (Jaccard index), and clustered the genomes using the Complete method. From module completeness, we created a Euclidean distance matrix, followed by clustering with the ward.D method. Based on module completeness clustering, we assigned genomes to metabolic Groups a- $\varepsilon$.

For each metabolic group, a representative metabolic map was created. Module completeness greater than $67 \%$ in at least half of the group members was considered representative of the group. Once the modules were selected, they were drawn and connected based on metabolic KEGG maps. Additional reaction, complexes, and transporters were added according to $\mathrm{KO}$ presence (e.g., aa. synthesis, oxidative phosphorylation complexes, flagellar motor).

For nitrogen metabolism, all relevant KOs were examined. For the purpose of this study, nitrate reduction was considered a separate path from denitrification/DNRA, since it could be the first step in both pathways, using the same enzymes. Denitrifying bacteria were considered to be bacteria capable of full conversion of nitrite to $\mathrm{N}_{2}$.
DNRA bacteria were considered to be bacteria capable of conversion of nitrite to ammonium using the nrfAH enzymes. No partial nitrogen process was considered for this paper, although it was present, according to perstep analysis.

\section{Supplementary information}

Supplementary information accompanies this paper at https://doi.org/10. 1186/s40168-020-0786-3.

\begin{abstract}
Additional file 1: Table S1. Genome parameters for the representative bacteria from the anammox bioreactor. Temporal strain shifts in the bioreactor. Analysis of shifts between bacterial strains during the first bioreactor crash and subsequent biomass amendment. Table S2. Genome parameters for the bacterial strains related to representative bacteria from the bioreactor. Table S3 assignment of genomes to different clusters. Figure S1. Analysis of community metabolism by gene presence. Figure S2. Reduced metabolic map of the Brocadia sp. Bacterium. Electron transfer pathways in the community. Analysis of different electron donors and acceptors utilized by the anammox community members. Central carbon metabolism in the community. Analysis of carbon utilization in the anammox microbial community. Table S4. Diversity indices of the anammox community. Table S5. Synthesis and transport of metabolites in the metabolic groups. Table S6. Confidence intervals of the Log-Ratio changes for each Reference Frame genome. Table S7. Closest relative based on distance in ML tree for anammox bacterial community. Figure S3. Maximum likelihood tree of the PVC clade (Planctomycetes, Verrucomicrobia, Chlamydiae, and Lentisphaerae). Figure S4. Schematic of the anaerobic membrane bioreactor Table S8. Composition of the synthetic wastewater feed. Supplemental methods. Refinement of the Candidatus Brocadia sp. genome. Table S9. Replication rates of genomes for each time point, calculated using the iRep program.
\end{abstract}

\section{Abbreviations}

AA: Anammox associated; Anammox: Anaerobic ammonium oxidation; ANI: Average nucleotide identity; Cl: Confidence interval; CPR: Candidate Phyla Radiation; D0: Day 0; D166: Day 166; D284: Day 284; D328: Day 328; D437: Day 437; D82: Day 82; DNRA: Dissimilatory nitrate reduction to ammonium; HMM: Hidden Markov Model; KEGG: Kyoto Encyclopedia of Genes and Genomes; KO: KEGG Orthology; LR: Log-ratio; nMDS: Nonmetric multidimensional scaling; NRR: Nitrogen removal rate; PN: Partial nitritation; RFg: Reference frame genome; SA: Source associated

\section{Acknowledgements}

The authors would like to thank Edmund Antell for his help with evaluating energy gains via nitrogen metabolism, as well as his feedback on manuscript revisions.

\section{Authors' contributions}

$K Y$ and $L Z$ supervised the study. $K Y, L Z, L A-C$, and DJ designed the study. $L Z$ built and maintained the bioreactor. RK analyzed metagenomics data and wrote the manuscript. JL analyzed $16 \mathrm{~S}$ rRNA gene data, analyzed bioreactor performance, and wrote the manuscript. JFB supervised the metagenomics analysis. WZ and KY contributed to bioreactor maintenance and analysis, sampling, and 165 rRNA gene data analysis. All authors read the manuscript and contributed with their input. All authors read and approved the final manuscript.

\section{Funding}

This research was supported by the National Natural Science Foundation of China (NO. 51709005 and NO. 51708362), and the National Science

Foundation through the Engineering Research Center for Relnventing the Nation's Water Infrastructure (ReNUWIt) ECC-1028962. This material is also based upon work supported by the National Science Foundation Graduate Research Fellowship under grant no. DGE 1106400. Any opinion, findings, and conclusions or recommendations expressed in this material are those of the authors and do not necessarily reflect the views of the National Science Foundation. 


\section{Availability of data and materials}

The metagenomic datasets analyzed during the current study are available on the National Center for Biotechnology Information (NCBI) Genbank, under project accession number PRJNA511011.

The 165 rRNA gene datasets analyzed during the current study are available on the Figshare repository: https://figshare.com/projects/Anammox Bioreactor/59324

The $\mathrm{R}$ script used to analyze the metagenomic data is also available on the Figshare repository, under the same link as above.

All other data generated during this study is either included in this published article (and its supplementary information files) or can be made available from the corresponding author upon reasonable request.

\section{Ethics approval and consent to participate}

Not applicable.

\section{Consent for publication}

Not applicable.

\section{Competing interests}

The authors declare that they have no competing interests.

\section{Author details}

${ }^{1}$ Department of Civil and Environmental Engineering, University of California Berkeley, Berkeley, CA, USA. ${ }^{2}$ Tighe \& Bond, Westwood, MA, USA. ${ }^{3}$ Department of Civil and Environmental Engineering, The University of Auckland, Auckland, New Zealand. ${ }^{4}$ Department of Earth and Planetary Sciences, University of California Berkeley, Berkeley, CA, USA. ${ }^{5}$ Earth and Environmental Sciences Division, Lawrence Berkeley National Laboratory, Berkeley, CA, USA. ${ }^{6}$ College of Chemistry and Environmental Engineering, Shenzhen University, Shenzhen, China. 'School of Environment and Energy, Shenzhen Graduate School, Peking University, Shenzhen, GD, China.

Received: 4 April 2019 Accepted: 7 January 2020

Published online: 24 January 2020

\section{References}

1. Mulder A, Graaf AA, Robertson LA, Kuenen JG. Anaerobic ammonium oxidation discovered in a denitrifying fluidized bed reactor. FEMS Microbiol Ecol. 1995;16:177-84

2. Kuenen JG. Anammox bacteria: from discovery to application. Nat Rev Microbiol. 2008;6:320-6.

3. Sonthiphand P, Hall MW, Neufeld JD. Biogeography of anaerobic ammoniaoxidizing (anammox) bacteria. Front Microbiol. 2014;5. https://doi.org/10. 3389/fmicb.2014.00399.

4. Connan R, Dabert P, Khalil H, Bridoux G, Béline F, Magrí A. Batch enrichment of anammox bacteria and study of the underlying microbial community dynamics. Chem Eng J. 2016;297:217-28.

5. Strous M, Pelletier E, Mangenot S, Rattei T, Lehner A, Taylor MW, et al. Deciphering the evolution and metabolism of an anammox bacterium from a community genome. Nature. 2006;440:790-4.

6. Kartal B, Maalcke WJ, de Almeida NM, Cirpus I, Gloerich J, Geerts W, et al. Molecular mechanism of anaerobic ammonium oxidation. Nature. 2011;479:127-30

7. Paques. Anammox sustainable nitrogen removal. https:/en.paques.nl/ mediadepot/1818a31cd232NEBbrochureAnammox.pdf. Accessed 26 Sept 2018.

8. Lackner S, Gilbert EM, Vlaeminck SE, Joss A, Horn H, van Loosdrecht MCM. Full-scale partial nitritation/anammox experiences - An application survey. Water Res. 2014;55:292-303.

9. Ali M, Okabe S. Anammox-based technologies for nitrogen removal: advances in process start-up and remaining issues. Chemosphere. 2015;141:144-53.

10. Jin R-C, Yang G-F, Yu J-J, Zheng P. The inhibition of the Anammox process: a review. Chem Eng J. 2012;197:67-79. https://doi.org/10.1016/j.cej.2012.05.014.

11. Li X, Klaus S, Bott C, He Z. Status, challenges, and perspectives of mainstream nitritation-anammox for wastewater treatment. Water Environ Res. 2018;90:634-49.

12. Li X-R, Du B, Fu H-X, Wang R-F, Shi J-H, Wang Y, et al. The bacterial diversity in an anaerobic ammonium-oxidizing (anammox) reactor community. Syst Appl Microbiol. 2009;32:278-89.

13. Gonzalez-Martinez A, Osorio F, Rodriguez-Sanchez A, Martinez-Toledo MV, Gonzalez-Lopez J, Lotti T, et al. Bacterial community structure of a lab-scale anammox membrane bioreactor. Biotechnol Prog. 2015;31:186-93.
14. Gonzalez-Martinez A, Osorio F, Morillo JA, Rodriguez-Sanchez A, Gonzalez-Lopez J, Abbas BA, et al. Comparison of bacterial diversity in full scale anammox bioreactors operated under different conditions. Biotechnol Prog. 2015;31:1464-72.

15. Gonzalez-Martinez A, Rodriguez-Sanchez A, Muñoz-Palazon B, Garcia-Ruiz MJ, Osorio F, van Loosdrecht MCM, et al. Microbial community analysis of a full-scale DEMON bioreactor. Bioprocess Biosyst Eng. 2015;38:499-508.

16. Pereira AD, Cabezas A, Etchebehere C, de Lemos Chernicharo CA, de Araújo JC. Microbial communities in anammox reactors: a review. Environ Technol Rev. 2017:6:74-93.

17. Bagchi S, Lamendella R, Strutt S, Van Loosdrecht MCM, Saikaly PE. Metatranscriptomics reveals the molecular mechanism of large granule formation in granular anammox reactor. Sci Rep. 2016;6:28327.

18. Speth DR, in 't M, Guerrero-Cruz S, Dutilh BE, Jetten MSM. Genome-based microbial ecology of anammox granules in a full-scale wastewater treatment system. Nat Commun. 2016;7:11172

19. Castro-Barros CM, Jia M, van Loosdrecht MCM, Volcke EIP, Winkler MKH. Evaluating the potential for dissimilatory nitrate reduction by anammox bacteria for municipal wastewater treatment. Bioresour Technol. 2017;233:363-72.

20. Lawson CE, Wu S, Bhattacharjee AS, Hamilton JJ, McMahon KD, Goel R, et al. Metabolic network analysis reveals microbial community interactions in anammox granules. Nat Commun. 2017:8:15416.

21. Tang X, Guo Y, Jiang B, Liu S. Metagenomic approaches to understanding bacterial communication during the anammox reactor start-up. Water Res, 2018;136:95-103.

22. van de Graaf AA, Mulder A, de Bruijn P, Jetten MS, Robertson LA, Kuenen $J G$. Anaerobic oxidation of ammonium is a biologically mediated process. Appl Environ Microbiol. 1995;61:1246-51.

23. Chen $\mathrm{H}, \mathrm{Yu}$ J-J, Jia X-Y, Jin R-C. Enhancement of anammox performance by $\mathrm{Cu}(\mathrm{III}, \mathrm{Ni}(\mathrm{II})$ and $\mathrm{Fe}(\mathrm{III})$ supplementation. Chemosphere. 2014;117:610-6.

24. Bi Z, Qiao S, Zhou J, Tang X, Zhang J. Fast start-up of Anammox process with appropriate ferrous iron concentration. Bioresour Technol. 2014;170:506-12.

25. Zhang X, Chen Z, Zhou Y, Ma Y, Ma C, Li Y, et al. Impacts of the heavy metals Cu (II), Zn (II) and Fe (II) on an Anammox system treating synthetic wastewater in low ammonia nitrogen and low temperature: Fe (II) makes a difference. Sci Total Environ. 2018;648:798-804.

26. Parks DH, Imelfort M, Skennerton CT, Hugenholtz P, Tyson GW. CheckM: assessing the quality of microbial genomes recovered from isolates, single cells, and metagenomes. Genome Res. 2015;25:1043-55.

27. Burstein D, Sun CL, Brown CT, Sharon I, Anantharaman K, Probst AJ, et al. Major bacterial lineages are essentially devoid of CRISPR-Cas viral defence systems. Nat Commun. 2016;7:10613.

28. Anantharaman K, Brown CT, Burstein D, Castelle CJ, Probst AJ, Thomas BC, et al. Analysis of five complete genome sequences for members of the class Peribacteria in the recently recognized Peregrinibacteria bacterial phylum. Peerf. 2016:4:e1607.

29. Brown CT, Hug LA, Thomas BC, Sharon I, Castelle CJ, Singh A, et al. Unusual biology across a group comprising more than $15 \%$ of domain Bacteria. Nature. 2015;523:208-11.

30. Anantharaman K, Brown CT, Hug LA, Sharon I, Castelle CJ, Probst AJ, et al. Thousands of microbial genomes shed light on interconnected biogeochemical processes in an aquifer system. Nat Commun. 2016;7:13219.

31. Zomorrodi AR, Segrè D. Genome-driven evolutionary game theory helps understand the rise of metabolic interdependencies in microbial communities. Nat Commun. 2017;8:1563.

32. Brown CT, Olm MR, Thomas BC, Banfield JF. Measurement of bacterial replication rates in microbial communities. Nat Biotechnol. 2016;34:1256-63.

33. Morton JT, Marotz C, Washburne A, Silverman J, Zaramela LS, Edlund A, et al. Establishing microbial composition measurement standards with reference frames. Nat Commun. 2019;10:1-11.

34. Strohm TO, Griffin B, Zumft WG, Schink B. Growth yields in bacterial denitrification and nitrate ammonification. Appl Environ Microbiol. 2007;73:1420-4.

35. Narita Y, Zhang L, Kimura Z-I, Ali M, Fujii T, Okabe S. Enrichment and physiological characterization of an anaerobic ammonium-oxidizing bacterium "Candidatus Brocadia sapporoensis.". Syst Appl Microbiol. 2017;40:448-57.

36. Probst AJ, Castelle CJ, Singh A, Brown CT, Anantharaman K, Sharon I, et al. Genomic resolution of a cold subsurface aquifer community provides metabolic insights for novel microbes adapted to high CO concentrations. Environ Microbiol. 2017;19:459-74.

37. High definition for systems biology of microbial communities: metagenomics gets genome-centric and strain-resolved. Curr Opin Biotechnol. 2016;39:174-81. 
38. Knelman JE, Nemergut DR. Changes in community assembly may shift the relationship between biodiversity and ecosystem function. Front Microbiol. 2014;5. https://doi.org/10.3389/fmicb.2014.00424

39. Fukami T, Dickie IA, Paula Wilkie J, Paulus BC, Park D, Roberts A, et al. Assembly history dictates ecosystem functioning: evidence from wood decomposer communities. Ecol Lett. 2010;13:675-84.

40. Johnston J, LaPara T, Behrens S. Composition and dynamics of the activated sludge microbiome during seasonal nitrification failure. Sci Rep. 2019;9:4565.

41. Ofiteru ID, Lunn M, Curtis TP, Wells GF, Criddle CS, Francis CA, et al. Combined niche and neutral effects in a microbial wastewater treatment community. Proceedings of the National Academy of Sciences. 2010;107: 15345-50. https://doi.org/10.1073/pnas.1000604107.

42. DeLong EF, Preston CM, Mincer T, Rich V, Hallam SJ, Frigaard N-U, et al. Community genomics among stratified microbial assemblages in the ocean's interior. Science. 2006;311:496-503.

43. Trivedi $P$, Anderson IC, Singh BK. Microbial modulators of soil carbon storage: integrating genomic and metabolic knowledge for global prediction. Trends Microbiol. 2013;21:641-51.

44. Lackner S, Terada A, Smets BF. Heterotrophic activity compromises autotrophic nitrogen removal in membrane-aerated biofilms: results of a modeling study. Water Res. 2008;42:1102-12.

45. Vanniel E, Arts P, Wesselink B, Robertson L, Kuenen J. Competition between heterotrophic and autotrophic nitrifiers for ammonia in chemostat cultures. FEMS Microbiology Letters. 1993;102:109-18. https://doi.org/10.1016/03781097(93)90006-n.

46. Brown EJ, Button DK, Lang DS. Competition between heterotrophic and autotrophic microplankton for dissolved nutrients. Microbial Ecology. 1981;7: 199-206. https://doi.org/10.1007/bf02010303.

47. Vet S, de Buyl S, Faust K, Danckaert J, Gonze D, Gelens L. Bistability in a system of two species interacting through mutualism as well as competition: Chemostat vs Lotka-Volterra equations. Plos One. 2018;13: e0197462. https://doi.org/10.1371/journal.pone.0197462.

48. Joshi NA FJN. Sickle: a sliding-window, adaptive, quality-based trimming tool for FastQ files (Version 1.33) [Software]. 2011. Available at https:// github.com/najoshi/sickle.

49. Peng Y, Leung HCM, Yiu SM, Chin FYL. IDBA-UD: a de novo assembler for single-cell and metagenomic sequencing data with highly uneven depth. Bioinformatics. 2012;28:1420-8.

50. Langmead B, Salzberg SL. Fast gapped-read alignment with Bowtie 2. Nat Methods. 2012:9:357-9.

51. Hyatt D, Chen G-L, Locascio PF, Land ML, Larimer FW, Hauser LJ. Prodigal: prokaryotic gene recognition and translation initiation site identification. BMC Bioinformatics. 2010;11:119.

52. Edgar RC. Search and clustering orders of magnitude faster than BLAST. Bioinformatics. 2010;26:2460-1.

53. Lowe TM, Eddy SR. tRNAscan-SE: a program for improved detection of transfer RNA genes in genomic sequence. Nucleic Acids Res. 1997;25:955-64.

54. Alneberg J, Bjarnason BS, de Bruijn I, Schirmer M, Quick J, ljaz UZ, et al. Binning metagenomic contigs by coverage and composition. Nat Methods. 2014;11:1144-6.

55. Wu Y-W, Simmons BA, Singer SW. MaxBin 2.0: an automated binning algorithm to recover genomes from multiple metagenomic datasets. Bioinformatics. 2015;32:605-7.

56. Sieber CMK, Probst AJ, Sharrar A, Thomas BC, Hess M, Tringe SG, et al. Recovery of genomes from metagenomes via a dereplication, aggregation, and scoring strategy. 2017. https://doi.org/10.1101/107789.

57. Hug LA, Thomas BC, Sharon I, Brown CT, Sharma R, Hettich RL, et al. Critical biogeochemical functions in the subsurface are associated with bacteria from new phyla and little studied lineages. Environ Microbiol. 2016;18:159-73.

58. Ultsch A, Mörchen F. ESOM-maps: Tools for clustering, visualization, and classification with emergent SOM; 2005.

59. Olm MR, Brown CT, Brooks B, Banfield JF. dRep: a tool for fast and accurate genomic comparisons that enables improved genome recovery from metagenomes through de-replication. ISME J. 2017;11:2864-8.

60. Hug LA, Baker BJ, Anantharaman K, Brown CT, Probst AJ, Castelle CJ, et al. A new view of the tree of life. Nat Microbiol. 2016;1:16048.

61. Matsen FA, Kodner RB, Armbrust EV. pplacer: linear time maximumlikelihood and Bayesian phylogenetic placement of sequences onto a fixed reference tree. BMC Bioinformatics. 2010;11:538.
62. Letunic I, Bork P. Interactive tree of life (iTOL) v3: an online tool for the display and annotation of phylogenetic and other trees. Nucleic Acids Res. 2016;44:W242-5.

63. Edgar RC. MUSCLE: multiple sequence alignment with high accuracy and high throughput. Nucleic Acids Res. 2004;32:1792-7.

64. Miller MA, Pfeiffer W, Schwartz T. Creating the CIPRES Science Gateway for inference of large phylogenetic trees. In: 2010 Gateway Computing Environments Workshop (GCE). p. 2010. https://doi.org/10.1109/gce.2010.5676129.

65. Paradis E, Claude J, Strimmer K. APE: analyses of phylogenetics and evolution in R language. Bioinformatics. 2004;20:289-90.

66. RStudio Team. RStudio: integrated development for R. Boston: RStudio, Inc;; 2015. http://www.rstudio.com/

67. R: The R Project for Statistical Computing. http://www.R-project.org. Accessed 26 Sept 2018.

68. Wu L, Wen C, Qin Y, Yin H, Tu Q, Van Nostrand JD, et al. Phasing amplicon sequencing on Illumina Miseq for robust environmental microbial community analysis. BMC Microbiol. 2015;15:125.

69. Schloss PD, Westcott SL, Ryabin T, Hall JR, Hartmann M, Hollister EB, et al. Introducing mothur: open-source, platform-independent, communitysupported software for describing and comparing microbial communities. Appl Environ Microbiol. 2009;75:7537-41.

70. Kozich JJ, Westcott SL, Baxter NT, Highlander SK, Schloss PD. Development of a dual-index sequencing strategy and curation pipeline for analyzing amplicon sequence data on the MiSeq Illumina sequencing platform. Appl Environ Microbiol. 2013;79:5112-20.

71. Pruesse E, Quast C, Knittel K, Fuchs BM, Ludwig W, Peplies J, et al. SILVA: a comprehensive online resource for quality checked and aligned ribosomal RNA sequence data compatible with ARB. Nucleic Acids Res. 2007;35:7188-96.

72. Evans J, Sheneman L, Foster J. Relaxed neighbor joining: a fast distancebased phylogenetic tree construction method. J Mol Evol. 2006;62:785-92.

73. Olm MR, Brown CT, Brooks B, Firek B, Baker R, Burstein D, et al. Identical bacterial populations colonize premature infant gut, skin, and oral microbiomes and exhibit different in situ growth rates. Genome Res. 2017; 27:601-12.

74. Moriya Y, Itoh M, Okuda S, Yoshizawa AC, Kanehisa M. KAAS: an automatic genome annotation and pathway reconstruction server. Nucleic Acids Res. 2007;35(Web Server issue):W182-5.

\section{Publisher's Note}

Springer Nature remains neutral with regard to jurisdictional claims in published maps and institutional affiliations.

Ready to submit your research? Choose BMC and benefit from:

- fast, convenient online submission

- thorough peer review by experienced researchers in your field

- rapid publication on acceptance

- support for research data, including large and complex data types

- gold Open Access which fosters wider collaboration and increased citations

- maximum visibility for your research: over $100 \mathrm{M}$ website views per year

At BMC, research is always in progress.

Learn more biomedcentral.com/submissions 